\title{
Highly Enantioselective Conjugate Addition of Malonate and $\beta$-Ketoester to Nitroalkenes: Asymmetric C-C Bond Formation with New Bifunctional Organic Catalysts Based on Cinchona Alkaloids
}

\author{
Hongming Li, Yi Wang, Liang Tang and Li Deng* \\ Department of Chemistry \\ Brandeis University \\ Waltham, Massachusetts 02454-9110
}

*To whom correspondence should be addressed

\section{Supporting Information}

General Information. ${ }^{1} \mathrm{H}$ and ${ }^{13} \mathrm{C}$ NMR spectra were recorded on a Varian instrument (400 $\mathrm{MHz}$ and $100 \mathrm{MHz}$, respectively) and internally referenced to tetramethylsilane signal or residual protio solvent signals. Data for ${ }^{1} \mathrm{H}$ NMR are recorded as follows: chemical shift $(\delta, \mathrm{ppm})$, multiplicity ( $\mathrm{s}$, singlet; $\mathrm{d}$, doublet; $\mathrm{t}$, triplet; q, quartet; $\mathrm{m}$, multiplet), intergration, coupling constant $(\mathrm{Hz})$. Data for ${ }^{13} \mathrm{C} \mathrm{NMR}$ are reported in terms of chemical shift $(\delta, \mathrm{ppm})$. Infrared spectra were recorded on a Perkin Elmer FT-IR Spectrometer and are reported in frequency of absorption. Low resolution mass spectra for all the new compounds done by either $20 \mathrm{eV}, \mathrm{CH}_{4} / \mathrm{CI}$ or $\mathrm{NH}_{3} / \mathrm{CI}$ were recorded on a Hewlett-Packard 5989A GC/MS, and exact mass spectra on a VG 7070 high resolution mass spectrometer. Specific rotations were measured on a Jasco Digital Polarimeter.

High pressure liquid chromatography (HPLC) analysis was performed on a HewlettPackard 1100 Series instrument equipped with a quaternary pump, using a Daicel Chiralcel OJ, OD Column (250 x $4.6 \mathrm{~mm})$ or Chiralpak AD Column $(250 \times 4.6 \mathrm{~mm})$. UV detection was monitored at $220 \mathrm{~nm}$ or at $215 \mathrm{~nm}$.

Nitroalkenes 5a, 5d, 5k, 5m were purchased from Aldrich Inc. and 5o was purchased from TCI America. They were used without other purifications. Other nitroalkenes were prepared according to literature procedures. ${ }^{1}$ 


\section{Structure of the catalysts}

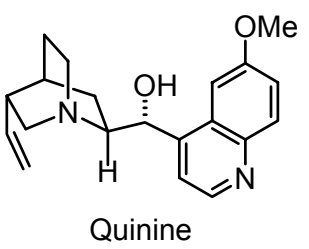<smiles>[R]O[C@H](c1ccnc2ccc(OC)cc12)[C@@H]1CN2CCCC1CC2</smiles>

DHQ-R<smiles>O=C1c2ccccc2C(=O)c2c(O)ccc(O)c21</smiles>

$(\mathrm{DHQD})_{2} \mathrm{AQN}$<smiles>C=CC1CC2CCN1C2[C@H](O)c1ccnc2ccc(OC)cc12</smiles><smiles>O=C(O[18OH])c1ccc(Cl)cc1</smiles>

DHQD-CLB<smiles>[O]c1nc(-c2ccccc2)nc([O-])c1-c1ccccc1</smiles>

$(\mathrm{DHQD})_{2} \mathrm{PYR}$<smiles>[R]OC(c1ccnc2ccc(OC)cc12)C1C2CN1CCC2CC</smiles><smiles>Nc1cccc2cc([OH2+])c3ccccc3c12</smiles><smiles>[O-]c1nnc(O)c2ccccc12</smiles>

$(\mathrm{DHQD})_{2} \mathrm{PHAL}$

Figure 1. The Structure of Cinchona Alkaloids

\section{Preparation of Quinidine 9-O-(9'-Phenanthryl) Ether, QD-PHN: ${ }^{2}$}

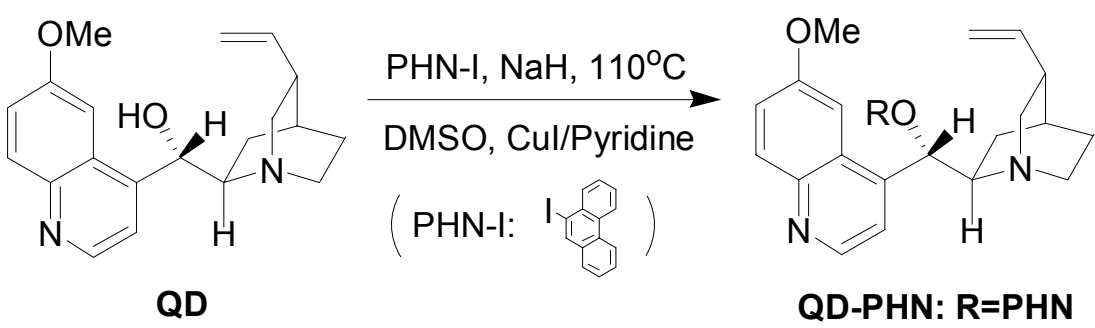

A three-necked round-bottomed flask $(500 \mathrm{~mL})$ equipped with a mechanical stirrer, reflux condenser, and inert gas in- and outlet was charged with quinidine (QD-1, 12.8g, $39.5 \mathrm{mmol}, 1.2 \mathrm{eq})$. The flask was flushed for $30 \mathrm{~min}$ with a gentle stream of argon. Anhydrous dimethyl sulfoxide $\left(130 \mathrm{~mL}\right.$, freshly distilled in the presence of $\mathrm{CaH}_{2}$ ) was added, and the reaction mixture was stirred at room temperature until all the quinidine was dissolved. Sodium hydride (60\% oil dispersion, $2.0 \mathrm{~g}, 1.5$ eq.) was added in small portions yielding an orange, slightly cloudy solution of the corresponding sodium alkoxide. Upon addition of pyridine $(6.4 \mathrm{~mL}, 2.4$ eq.) and copper(I) iodide(7.8 g, 1.2 eq.) to the reaction mixture at room temperature, the color of the reaction mixture was changed from orange to dark green. After $30 \mathrm{~min}$ all of the precipitate dissolved, and a clear solution was formed. 9-iodophenanthrene $(10.0 \mathrm{~g}, 32.9 \mathrm{mmol})$, was added, and the reaction mixture was kept at $113^{\circ} \mathrm{C}$ for $70 \mathrm{~h}$ (oil bath, temperature: $120^{\circ} \mathrm{C}$ ). The reaction 
mixture was allowed to cool to room temperature. Water (100 mL), methylene chloride $(100 \mathrm{~mL})$, and diethyl ether $(100 \mathrm{~mL})$ were successively added to the brown solution followed by ethylenediaminetetraacetate disodium salt dihydrate $(20 \mathrm{~g})$ and concentrated aqueous ammonia solution $(20 \mathrm{~mL}, 29 \%$, w/w). The argon inlet was removed, and a gentle stream of air was flushed through the well-agitated reaction mixture for about $1 \mathrm{~h}$. The reaction mixture was transferred to a separatory funnel and the turquoise blue aqueous phase separated from the dark brown organic phase. The aqueous layer was washed twice with methylene chloride $(100 \mathrm{~mL})$, and the combined organic phases were extracted three times with aqueous ammonia solution $(200 \mathrm{~mL}, 5 \%$, w/w $)$ until the aqueous phase remained colorless. Then the organic layer was washed with aqueous $\mathrm{HCl}(1.0 \mathrm{~N}, 2 \times 50 \mathrm{~mL})$ twice, with $\mathrm{H}_{2} \mathrm{O}$ three times $(3 \times 50 \mathrm{~mL})$ to remove the excess QD. The organic layer was washed with $\mathrm{NH}_{4} \mathrm{OH}$ and dried over $\mathrm{Na}_{2} \mathrm{SO}_{4}$ and the solvent was removed in vacuo. The residue was dissolved in $\mathrm{Et}_{2} \mathrm{O}(300 \mathrm{~mL})$ and treated with $\mathrm{HCl}\left(1 \mathrm{~N}\right.$ in $\left.\mathrm{Et}_{2} \mathrm{O}\right)$ until no further precipitates was generated. The precipitates were collected and dissolved in $\mathrm{CH}_{2} \mathrm{Cl}_{2}$ and basified with saturated aqueous $\mathrm{NH}_{4} \mathrm{OH}$. The organic phase was washed with brine, dried over $\mathrm{Na}_{2} \mathrm{SO}_{4}$. The solvent was removed in vacuo to furnish QD-PHN as a yellowish foam $\left(5.6 \mathrm{~g}, 66 \%\right.$ yield). $[\alpha]_{\mathrm{D}}{ }^{25}=+310.7$ (c $0.89 \mathrm{EtOH}) ;{ }^{1} \mathrm{H} \mathrm{NMR}\left(\mathrm{CDCl}_{3}, 400 \mathrm{MHz}\right) \delta 8.65-8.71(\mathrm{~m}, 2 \mathrm{H}), 8.61(\mathrm{~d}, J=4.8 \mathrm{~Hz}, 1 \mathrm{H})$, $8.52(\mathrm{~d}, J=8 \mathrm{~Hz}, 1 \mathrm{H}), 8.07(\mathrm{~d}, J=9.2 \mathrm{~Hz}, 1 \mathrm{H}), 7.70-7.75(\mathrm{~m}, 2 \mathrm{H}), 7.55(\mathrm{~d}, J=2.4 \mathrm{~Hz}$, $1 \mathrm{H}), 7.38-7.46(\mathrm{~m}, 5 \mathrm{H}), 6.66(\mathrm{~s}, 1 \mathrm{H}), 6.35(\mathrm{br}, 1 \mathrm{H}), 6.12-6.21(\mathrm{~m}, 1 \mathrm{H}), 5.18(\mathrm{~d}, J=10.4$ $\mathrm{Hz}, 1 \mathrm{H}), 5.12(\mathrm{~m}, 1 \mathrm{H}), 4.03(\mathrm{~s}, 3 \mathrm{H}), 3.32-3.42(\mathrm{~m}, 2 \mathrm{H}), 2.97-3.06(\mathrm{~m}, 2 \mathrm{H}), 2.79-2.87(\mathrm{~m}$, $1 \mathrm{H}), 2.44-2.50(\mathrm{t}, J=10.0 \mathrm{~Hz}, 1 \mathrm{H}), 2.34-3.25(\mathrm{~m}, 1 \mathrm{H}), 1.97(\mathrm{br}, 1 \mathrm{H}), 1.55-1.62(\mathrm{~m}, 3 \mathrm{H})$; ${ }^{13} \mathrm{C} \mathrm{NMR}\left(\mathrm{CDCl}_{3}, 100 \mathrm{MHz}\right): \delta 158.1,150.4,147.71,144.7,143.7,140.3,132.3,132.2$, $131.5,127.5,127.3,126.8,126.7,126.6,126.4,124.5,122.8,122.7,122.3,121.8,118.2$, $114.7,104.8,100.8,78.8,60.5,55.8,50.2,49.9,39.6,27.8,26.5,22.1 ; \mathrm{IR}\left(\mathrm{CHCl}_{3}\right) v$ 3062, 2935, 2863, 1622, 1594, 1507, 1454, 1226, 1117, 750; HRMS (EI) m/z calcd for $\left(\mathrm{C}_{34} \mathrm{H}_{32} \mathrm{~N}_{2} \mathrm{O}_{2}{ }^{+}\right)$: 500.2464, found: 500.2481.

\section{Preparation of Quinidine 9-O-Benzyl Ether, QD-OBn:}
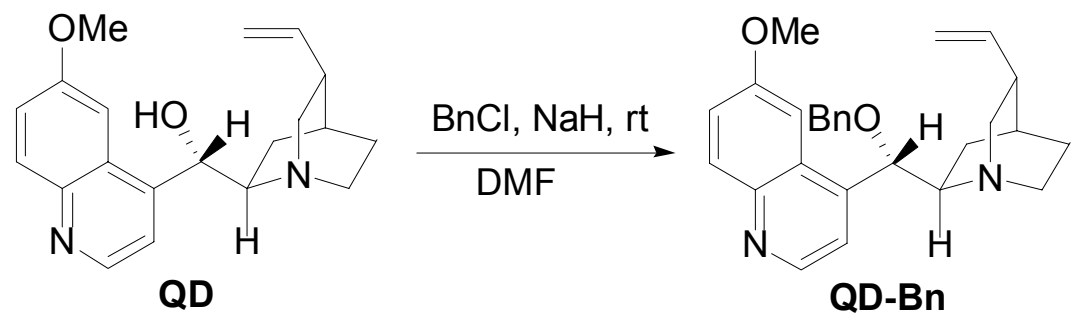

To the solution of QD $(2.0 \mathrm{~g}, 6.2 \mathrm{mmol})$ in DMF $(20 \mathrm{~mL}$, freshly distilled from a suspension of $\mathrm{CaH}_{2}$ in DMF) was added $\mathrm{NaH}(0.68 \mathrm{~g}, 57 \%$ suspension in mineral oil, 2.5 eq.). The resulting mixture was stitted at room temperature for $2 \mathrm{~h}$. Then $\mathrm{BnCl}(0.78 \mathrm{~mL}$, 1.1 eq.) was added dropwisely via a syringe in 10 minutes. The resulting mixture was stirred overnight. When the reaction was completed, brine $(20 \mathrm{~mL})$ was added carefully and the resulting mixture was extracted with EtOAc $(100 \mathrm{~mL})$. The organic phase was washed with brine $(3 \times 50 \mathrm{~mL})$, dried over $\mathrm{Na}_{2} \mathrm{SO}_{4}$, and concentrated in vacuo. The residue was purified by flash chromatography $(\mathrm{MeOH} / \mathrm{EtOAc:} 1 / 40)$ to give a yellowish oil $(2.3 \mathrm{~g}, 90 \%$ yield $) .[\alpha]_{\mathrm{D}}{ }^{25}=+137.4\left(\mathrm{c} 0.86 \mathrm{CHCl}_{3}\right) ;{ }^{1} \mathrm{H}$ NMR $\left(400 \mathrm{MHz}, \mathrm{CDCl}_{3}\right) \delta$ 
$8.77(\mathrm{~d}, J=5.2 \mathrm{~Hz}, 1 \mathrm{H}), 8.05(\mathrm{~d}, J=9.2 \mathrm{~Hz}, 1 \mathrm{H}), 7.49(\mathrm{~d}, J=4.0 \mathrm{~Hz}, 1 \mathrm{H}), 7.40-7.31(\mathrm{~m}$, 7H), 6.00-5.92 (m, 1H), 5.23 (br, 1H), 5.02-5.00 (m, 1H), $4.97(\mathrm{~s}, 1 \mathrm{H}), 4.49-4.37$ (AB, $2 \mathrm{H}), 3.90(\mathrm{~s}, 3 \mathrm{H}), 3.26(\mathrm{~m}, 1 \mathrm{H}), 3.09-3.08(\mathrm{~m}, 1 \mathrm{H}), 2.93-2.71(\mathrm{~m}, 3 \mathrm{H}), 2.26-2.22(\mathrm{~m}, 1 \mathrm{H})$, 2.11-2.05 (m, 1H), $1.75(\mathrm{br}, 1 \mathrm{H}), 1.51-1.43(\mathrm{~m}, 2 \mathrm{H}), 1.29-1.24(\mathrm{~m}, 1 \mathrm{H}) ;{ }^{13} \mathrm{C} \mathrm{NMR}(100$ $\left.\mathrm{MHz} \mathrm{CDCl}_{3}\right) \delta 157.7,147.6,144.8,144.7,140.7,137.8,131.9,128.3,127.9,127.7$, $127.5,121.7,119.1,114.3,101.2,80.9,71.2,60.1,55.5,50.1,49.5,40.1,28.1,26.6,22.4$; IR $\left(\mathrm{CHCl}_{3}\right) \vee 2935,2869,1620,1589,1507,1240,1029$.

\section{General Procedure for the Preparation of QD-4a, QD-4b, QD-4c and Q-4a: ${ }^{3}$}

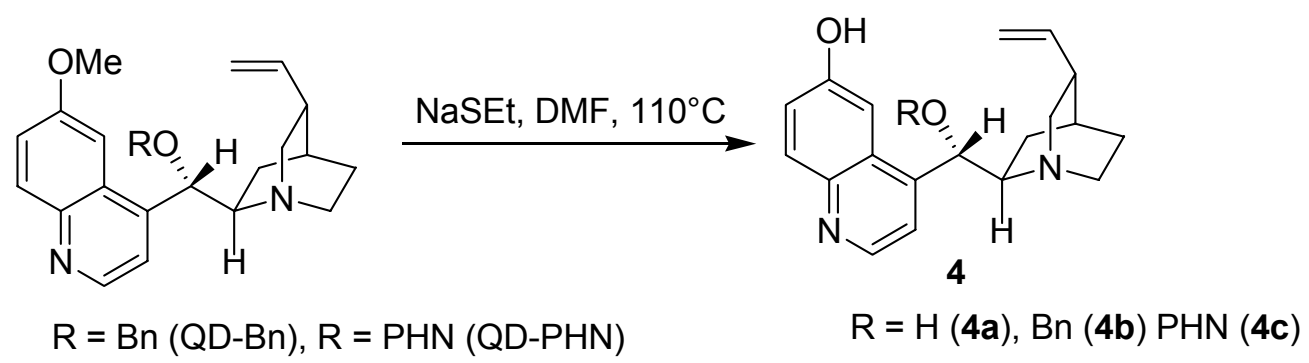

Under $\mathrm{N}_{2}$ atmosphere, a suspension of the starting cinchona alkaloid derivative (8.0 mmol) and NaSEt (4 equiv.) in dry DMF $(50 \mathrm{ml}$, freshly distilled from the suspension of $\mathrm{CaH}_{2}$ in DMF) was stirred at $110{ }^{\circ} \mathrm{C}$ until a TLC analysis showed that the starting material was completely consumed (4-6 hours). The reaction mixture was cooled down to room temperature, mixed with sat. $\mathrm{NH}_{4} \mathrm{Cl}(40 \mathrm{ml})$ and $\mathrm{H}_{2} \mathrm{O}(50 \mathrm{ml})$. The $\mathrm{pH}$ value of the solution was determined to be around 7. The resulting mixture was extracted with EtOAc $(2 \times 200 \mathrm{~mL})$. The organic phase was washed with brine $(4 \times 50 \mathrm{ml})$, dried over $\mathrm{Na}_{2} \mathrm{SO}_{4}$, and concentrated in vacuo. The residue was subjected to flash chromatography to afford the desired product.

QD-4a was obtained as a yellowish solid in $92 \%$ yield from quinidine after flash chromatography (elution gradient: Ethyl Acetate/MeOH $\left./ \mathrm{Et}_{3} \mathrm{~N}=100 / 2 / 3\right) .[\alpha]_{\mathrm{D}}{ }^{25}=+$ 240.3 (c 1.13, EtOH); ${ }^{1} \mathrm{H}$ NMR (400 MHz, CD $\left.3 \mathrm{OD}\right) \delta 8.58$ (d, J=4.4 Hz, $\left.1 \mathrm{H}\right), 7.88$ (d, $J=9.2 \mathrm{~Hz}, 1 \mathrm{H}), 7.62(\mathrm{~d}, J=5.2 \mathrm{~Hz}, 1 \mathrm{H}), 7.31(\mathrm{dd}, J=2.4 \mathrm{~Hz}, 9.2 \mathrm{~Hz}, 1 \mathrm{H}), 7.25(\mathrm{~d}, J$ $=2.4 \mathrm{~Hz}, 1 \mathrm{H}), 6.10-6.19(\mathrm{~m}, 1 \mathrm{H}), 5.58(\mathrm{~d}, J=2.8 \mathrm{~Hz}, 1 \mathrm{H}), 5.10(\mathrm{~d}, J=18.8 \mathrm{~Hz}, 1 \mathrm{H})$, $5.06(\mathrm{~d}, J=10.4 \mathrm{~Hz}, 1 \mathrm{H}), 3.60(\mathrm{ddd}, J=2.0 \mathrm{~Hz}, 8.0 \mathrm{~Hz}, 13.6 \mathrm{~Hz}, 1 \mathrm{H}), 3.03(\mathrm{dt}, J=2.4$ $\mathrm{Hz}, 9.2 \mathrm{~Hz}, 1 \mathrm{H}), 2.87-2.94(\mathrm{~m}, 2 \mathrm{H}), 2.74-2.82(\mathrm{~m}, 1 \mathrm{H}), 2.30(\mathrm{dt}, J=8.8 \mathrm{~Hz}, 8.4 \mathrm{~Hz}, 1 \mathrm{H})$, 2.18-2.23 (m, 1H), 1.71 ( br, $1 \mathrm{H}), 1.50-1.58(\mathrm{~m}, 2 \mathrm{H}), 1.00-1.07(\mathrm{~m}, 1 \mathrm{H}) ;{ }^{13} \mathrm{C} \mathrm{NMR}(100$ $\left.\mathrm{MHz}, \mathrm{CD}_{3} \mathrm{OD}\right) \delta 158.0,149.8,147.4,143.9,141.6,131.4,128.4,123.3,119.7,115.2$, $105.1,72.2,60.6,50.8,50.4,41.3,29.7,27.0,21.2$; IR $\left(\mathrm{CHCl}_{3}\right) \vee 3062,2929,2300-$ 3500 (br), 1616, 1505, 1229, $739 \mathrm{~cm}^{-1}$; HRMS (FAB) m/z calcd for $\left(\mathrm{C}_{19} \mathrm{H}_{22} \mathrm{~N}_{2} \mathrm{O}_{2}+\mathrm{H}^{+}\right)$: 311.1760, found: 311.1755 .

QD-4b was obtained as a yellowish powder in $87 \%$ yield from QD-Bn after flash chromatography (elution gradient: Ethyl Acetate $\mathrm{MeOH}=50 / 1$ to $10 / 1$ ). $[\alpha]_{\mathrm{D}}{ }^{25}=154.7$ $\left(\mathrm{c} 1.05, \mathrm{CHCl}_{3}\right) ;{ }^{1} \mathrm{H} \mathrm{NMR}\left(400 \mathrm{MHz}, \mathrm{CDCl}_{3}\right) \delta 10.92(\mathrm{br}, 1 \mathrm{H}), 8.67(\mathrm{~d}, J=4.4 \mathrm{~Hz}, 1 \mathrm{H})$, 8.01(d, $J=9.2 \mathrm{~Hz}, 1 \mathrm{H}), 7.86(\mathrm{~s}, 1 \mathrm{H}), 7.44(\mathrm{br}, 1 \mathrm{H}), 7.35(\mathrm{dd}, J=2.4 \mathrm{~Hz}, 8.8 \mathrm{~Hz}, 1 \mathrm{H})$, 7.24-7.28 (m, 5H), 5.97-5.89 (m, 1H), 5.53 (br, 1H), $4.97(\mathrm{~d}, J=10.0 \mathrm{~Hz}, 1 \mathrm{H}), 4.94(\mathrm{~s}$, 
$1 \mathrm{H}), 4.34-4.25$ (AB, 2H), 3.56 (br, 1H),3.05-3.03 (m, 2H), 2.87-2.78 (m, 2H), 2.26-2.24 (m, 2H), $1.74(\mathrm{br}, 1 \mathrm{H}), 1.52-1.39(\mathrm{~m}, 2 \mathrm{H}), 1.13(\mathrm{br}, 1 \mathrm{H}) ;{ }^{13} \mathrm{C} \mathrm{NMR}\left(100 \mathrm{MHz}, \mathrm{CDCl}_{3}\right) \delta$ 157.0, 146.5, 143.6, 139.9, 137.7, 131.3, 128.3, 127.9, 127.8, 127.7, 127.6, 123.4, 114.9, 106.7, 79.0, 71.2, 59.0, 49.7, 49.2, 39.6, 28.0, 25.9. IR $\left(\mathrm{CHCl}_{3}\right) \vee 2400-3500$ (br.), 2939, $2873,1617,1590,1511,1468,1240,1068,750 \mathrm{~cm}^{-1}$; HRMS (ESI) $\mathrm{m} / \mathrm{z}$ calcd for $\left(\mathrm{C}_{26} \mathrm{H}_{28} \mathrm{~N}_{2} \mathrm{O}_{2}+\mathrm{H}^{+}\right): 401.2229$, found: 401.2229 .

QD-4c was obtained as a yellowish solid in 91\% yield from QD-PHN after flash chromatography (elution gradient: Ethyl Acetate/ $\mathrm{MeOH}=20 / 1) .[\alpha]_{\mathrm{D}}{ }^{25}=-304.6$ (c 0.98, $\left.\mathrm{CHCl}_{3}\right) ;{ }^{1} \mathrm{H} \mathrm{NMR}\left(400 \mathrm{MHz}, \mathrm{CDCl}_{3}\right) \delta 8.69-8.67(\mathrm{~m}, 1 \mathrm{H}), 8.63-8.61(\mathrm{~m}, 1 \mathrm{H}), 8,46(\mathrm{~d}, J$ $=8.0 \mathrm{~Hz}, 1 \mathrm{H}), 8.20(\mathrm{~d}, 1 \mathrm{H}, J=2.0 \mathrm{~Hz}, 1 \mathrm{H}), 7.78(\mathrm{~d}, J=9.2 \mathrm{~Hz}, 1 \mathrm{H}), 7.74-7.70(\mathrm{~m}, 2 \mathrm{H})$, $7.35(\mathrm{~d}, J=5.2 \mathrm{~Hz}, 1 \mathrm{H}), 7.25-7.26(\mathrm{~m}, 1 \mathrm{H}), 7.20(\mathrm{t}, J=7.2 \mathrm{~Hz}, 1 \mathrm{H}), 6.80(\mathrm{t}, J=7.2 \mathrm{~Hz}$, $1 \mathrm{H}), 6.64(\mathrm{~s}, 1 \mathrm{H}), 6.50(\mathrm{~d}, J=7.2 \mathrm{~Hz}, 1 \mathrm{H}), 6.33(\mathrm{~s}, 1 \mathrm{H}), 6.23-6.15(\mathrm{~m}, 1 \mathrm{H}), 5.27$ (d, $J=$ $14.4 \mathrm{~Hz}, 1 \mathrm{H}), 5.12(\mathrm{~d}, J=17.2 \mathrm{~Hz}, 1 \mathrm{H}), 3.58-3.53(\mathrm{~m}, 1 \mathrm{H}), 3.36(\mathrm{t}, J=9.2 \mathrm{~Hz}, 1 \mathrm{H}), 3.09-$ $2.99(\mathrm{~m}, 2 \mathrm{H}), 2.79-2.68(\mathrm{~m}, 2 \mathrm{H}), 2.30-2.28(\mathrm{~m}, 1 \mathrm{H}), 2.00(\mathrm{br}, 1 \mathrm{H}), 1.54-1.52(\mathrm{~m}, 2 \mathrm{H})$, 1.43-1.34 (m, 1H); ${ }^{13} \mathrm{C}$ NMR $\left(100 \mathrm{MHz}, \mathrm{CDCl}_{3}\right) \delta 157.0,149.6,146.9,143.7,142.4$, $139.4,131.9,131.8,131.5,127.3,127.2,127.1,126.6,126.4,126.3,126.1,124.5,123.4$, $122.8,122.6,121.9,117.5,115.3,106.1,105.0,76.8,59.2,49.7,49.5,38.8,27.4,25.7$, 20.3; IR $\left(\mathrm{CHCl}_{3}\right) \vee 3300-2800$ (br), 3070, 2939, 2870, 1622, 1506, 1455, 1225, 1115, $753 \mathrm{~cm}^{-1}$; HRMS (ESI) $\mathrm{m} / \mathrm{z}$ calcd for $\left(\mathrm{C}_{33} \mathrm{H}_{30} \mathrm{~N}_{2} \mathrm{O}_{2}+\mathrm{H}^{+}\right): 487.2386$, found: 487.2370 .

Q-4a was obtained as a white solid in $80 \%$ yield from quinine after flash chromatography (elution grdient: Ethyl Acetate/ $\left.\mathrm{MeOH} / \mathrm{Et}_{3} \mathrm{~N}=100 / 2 / 3\right) .[\alpha]_{\mathrm{D}}{ }^{25}=-162.8$ (c $0.93, \mathrm{EtOH}$ ); ${ }^{1} \mathrm{H}$ NMR $\left(400 \mathrm{MHz}, \mathrm{CD}_{3} \mathrm{OD}\right) \delta 8.58(\mathrm{~d}, J=4.8 \mathrm{~Hz}, 1 \mathrm{H}), 7.89(\mathrm{~d}, J=9.2 \mathrm{~Hz}, 1 \mathrm{H}), 7.61(\mathrm{~d}$, $J=4.8 \mathrm{~Hz}, 1 \mathrm{H}), 7.32$ ( dd, $J=2.4 \mathrm{~Hz}, 9.2 \mathrm{~Hz}, 1 \mathrm{H}), 7.28(\mathrm{~d}, J=2.4 \mathrm{~Hz}, 1 \mathrm{H}), 5.69-5.78$ (m, 1H), $5.51(\mathrm{~d}, J=3.2 \mathrm{~Hz}, 1 \mathrm{H}), 4.95(\mathrm{~d}, J=17.2 \mathrm{~Hz}, 1 \mathrm{H}), 4.88(\mathrm{~d}, J=9.2 \mathrm{~Hz}, 1 \mathrm{H})$, 3.66-3.73 (m, 1H), 3.05-3.13 (m, 2H), 2.63-2.74 (m, 2H), 2.34 (br, $1 \mathrm{H}), 1.80-1.89(\mathrm{~m}$, $2 \mathrm{H}), 1.77-1.78(\mathrm{~m}, 1 \mathrm{H}), 1.54-1.61(\mathrm{~m}, 1 \mathrm{H}), 1.39-1.46(\mathrm{~m}, 1 \mathrm{H}) ;{ }^{13} \mathrm{C}$ NMR $(100 \mathrm{MHz}$, $\left.\mathrm{CD}_{3} \mathrm{OD}\right) \delta 157.9,149.8,147.4,144.0,142.6,131.4,128.4,123.3,119.8,115.0,105.1$, 72.2, 60.9, 57.6, 48.4, 44.2, 40.9, 29.2, 28.1, 21.8; IR $\left(\mathrm{CHCl}_{3}\right)$ v 3055, 2937, 2870, 23003500 (br), 1616, 1457, 1236, $1067 \mathrm{~cm}^{-1}$; HRMS (FAB) m/z calcd. for $\left(\mathrm{C}_{19} \mathrm{H}_{22} \mathrm{~N}_{2} \mathrm{O}_{2}+\mathrm{H}^{+}\right)$: 311.1760, found: 311.1761 .

Preparation of Racemic adducts (6a-r): At room temperature to a solution of a nitroalkene $(0.1 \mathrm{mmol})$ in THF $(1.0 \mathrm{M})$ was added dimethyl malonate $(34 \mu \mathrm{L}, 3$ eq.) and base [for aryl substituted 5a-5o, DABCO (20 mol\%) was used; for alkyl substituted 5p-r, $\mathrm{KOBu}^{\mathrm{t}}(5 \mathrm{~mol} \%)$ was used]. After the reaction went to completion, the reaction mixture was diluted with ethyl acetate, washed with $\mathrm{HCl}(1.0 \mathrm{~N}$, aq.) and brine. The organic layer was dried over $\mathrm{Na}_{2} \mathrm{SO}_{4}$ and concentrated in vacuo. The residue was purified by flash chromatography.

General procedure for enantioselective Michael addition of dimethyl malonate to nitroalkenes: 


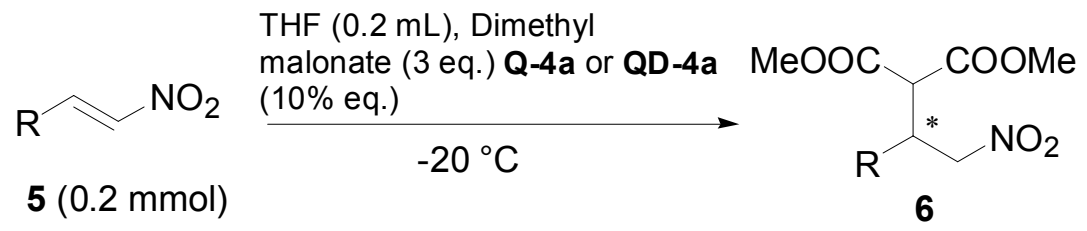

At $-20^{\circ} \mathrm{C}$, to a solution of a nitroalkene $(0.2 \mathrm{mmol})$ in THF $(0.2 \mathrm{~mL})$ was added dimethyl malonate ( $68 \mu \mathrm{L}, 3$ eq.) and the chiral catalyst $(6.2 \mathrm{mg}, 10 \mathrm{~mol} \%$.). The resulting mixture was kept at $-20{ }^{\circ} \mathrm{C}$ until $\mathbf{5}$ is consumed. The reaction mixture was then passed through a plug of silica gel for the removal of the catalyst (eluent: ether or ethyl acetate). The filtrate was concentrated in vacuo and the residue was purified by flash chromatography.<smiles>COC(=O)C(C(C)=O)[C@H](C[N+](=O)[O-])c1ccccc1</smiles>

(S)-(+)-6a

(S)-(+)-Methyl 2-carbomethoxy-4-nitro-3-phenyl-butyrate (S)$(+)-6 a$. This product was obtained as a colorless oil in $97 \%$ yield after flash chromatography (elution grdient: Ethyl Acetate / Hexane $=1 / 15$ to $1 / 10$ ) and $96 \%$ ee as determined by HPLC analysis [Daicel chiralcel OD, Hexanes:IPA, 70:30, $0.9 \mathrm{ml} / \mathrm{min}, \lambda 220 \mathrm{~nm}, \mathrm{t}$ (major) $=$ $11.6 \mathrm{~min}, \mathrm{t}($ minor $)=13.7 \mathrm{~min}]$ from a reaction catalyzed by $\mathbf{Q}-\mathbf{4 a}$ $(10 \mathrm{~mol} \%)$ at $-20^{\circ} \mathrm{C}$ for 36 hours. $[\alpha]_{\mathrm{D}}{ }^{25}=+5.9\left(\mathrm{c} 1.02, \mathrm{CHCl}_{3}\right) ;{ }^{1} \mathrm{H}$ NMR (400 MHz, $\left.\mathrm{CDCl}_{3}\right) \delta$ 7.34-7.22 (m, 5H), 4.95-4.85 (m, 2H), $4.25(\mathrm{dt}, J=5.2 \mathrm{~Hz}$, $8.8 \mathrm{~Hz}, 1 \mathrm{H}), 3.86(\mathrm{~d}, J=9.2 \mathrm{~Hz}, 1 \mathrm{H}), 3.77(\mathrm{~s}, 3 \mathrm{H}), 3.57(\mathrm{~s}, 3 \mathrm{H}) ;{ }^{13} \mathrm{C}$ NMR $(100 \mathrm{MHz}$, $\left.\mathrm{CDCl}_{3}\right) \delta 167.8,167.2,136.1,128.9,128.3,127.8,77.3,54.7,52.9,52.7,42.9$; IR $\left(\mathrm{CHCl}_{3}\right)$ v 3034, 2957, 1732, 1557, 1435, $1259 \mathrm{~cm}^{-1}$; HRMS (CI) $\mathrm{m} / \mathrm{z}$ calcd. for $\left(\mathrm{C}_{13} \mathrm{H}_{15} \mathrm{NO}_{6}+\mathrm{H}^{+}\right)$282.0964, found 282.0969.

The absolute configuration of (+)-6a was determined to be $S$ isomer by comparing the specific optical rotation with literature value ${ }^{4} \cdot[\alpha]_{\mathrm{D}}{ }^{25}=+5.9\left(\mathrm{c} 1.02, \mathrm{CHCl}_{3}\right)$ for $96 \%$ ee $\left[\right.$ lit. $[\alpha]_{\mathrm{D}}^{25}=+4.40\left(\mathrm{c} 1.02, \mathrm{CHCl}_{3}\right) 93 \%$ ee $]$.

(-)-Methyl 2-carbomethoxy-4-nitro-3-phenyl-butyrate R-(-)-6a. This product was obtained as a colorless oil in $99 \%$ yield and $93 \%$ ee from a reaction catalyzed by QD-4a $(10 \mathrm{~mol} \%)$ at $-20^{\circ} \mathrm{C}$ for 36 hours.

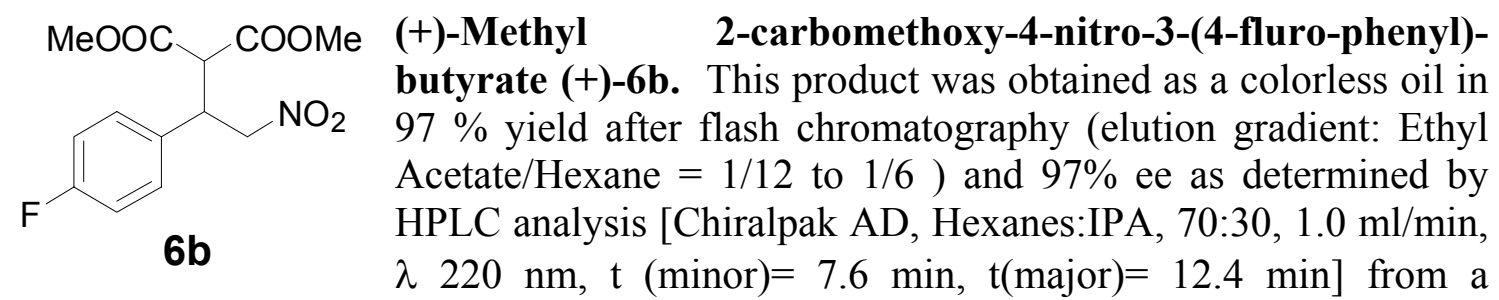

reaction catalyzed by Q-4a $(10 \mathrm{~mol} \%)$ at $-20^{\circ} \mathrm{C}$ for 36 hours. $[\alpha]_{\mathrm{D}}{ }^{25}=+6.2(\mathrm{c} 1.04$, $\left.\mathrm{CHCl}_{3}\right) ;{ }^{1} \mathrm{H}$ NMR $\left(400 \mathrm{MHz}, \mathrm{CDCl}_{3}\right) \delta 7.22(\mathrm{dd}, J=4.8 \mathrm{~Hz}, 8.4 \mathrm{~Hz}, 2 \mathrm{H}), 7.02(\mathrm{t}, J=8.4$ $\mathrm{Hz}, 2 \mathrm{H}), 4.91(\mathrm{dd}, J=4.8 \mathrm{~Hz}, 12.8 \mathrm{~Hz}, 1 \mathrm{H}), 4.84(\mathrm{dd}, J=9.2 \mathrm{~Hz}, 12.8 \mathrm{~Hz}, 1 \mathrm{H}), 4.24$ (dt, $J=5.6 \mathrm{~Hz}, 9.2 \mathrm{~Hz}, 1 \mathrm{H}), 3.82(\mathrm{~d}, J=9.2 \mathrm{~Hz}, 1 \mathrm{H}), 3.77(\mathrm{~s}, 3 \mathrm{H}), 3.58(\mathrm{~s}, 3 \mathrm{H}) ;{ }^{13} \mathrm{C} \mathrm{NMR}$ $\left(100 \mathrm{MHz}, \mathrm{CDCl}_{3}\right) \delta$ 167.6, 167.1, 163.7, 161.2, 131.84, 131.80, 129.68, 129.60, 116.1, 115.9, 77.4, 54.6, 53.0, 52.9, 42.2; IR $\left(\mathrm{CHCl}_{3}\right) \vee 3011,2958,2915,1733,1606,1557$, 
1436, $1231 \mathrm{~cm}^{-1}$; HRMS (CI) $\mathrm{m} / \mathrm{z}$ calcd. for $\left(\mathrm{C}_{13} \mathrm{H}_{14} \mathrm{FNO}_{6}+\mathrm{H}^{+}\right): 300.0883$, found 300.0886 .

(-)-Methyl 2-carbomethoxy-4-nitro-3-(4-fluro-phenyl)-butyrate (-)-6b. This product was obtained as a colorless oil in $97 \%$ yield and $94 \%$ ee from a reaction catalyzed by QD-4a (10 mol \%) at $-20^{\circ} \mathrm{C}$ for 36 hours.

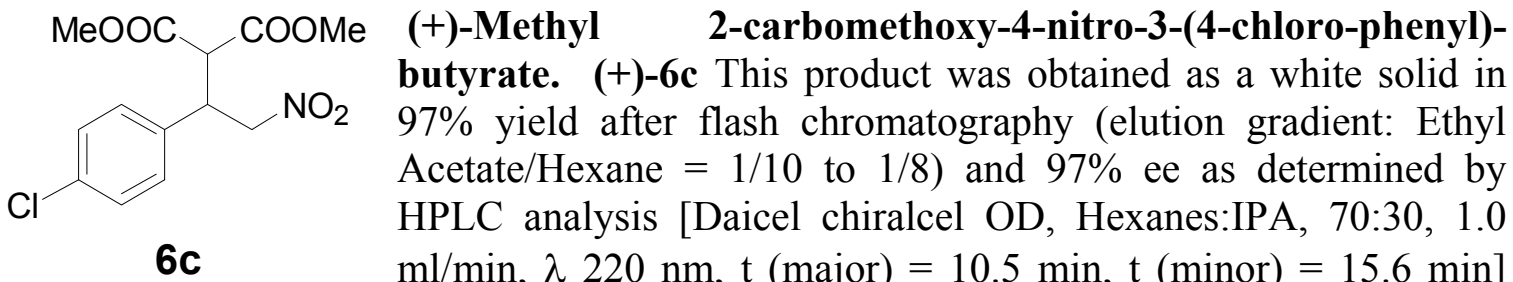
from a reaction catalyzed by $\mathbf{Q}-4 \mathbf{a}(10 \mathrm{~mol} \%)$ at $-20^{\circ} \mathrm{C}$ for 36 hours. $[\alpha]_{\mathrm{D}}{ }^{25}=+7.5(\mathrm{c}$ $\left.1.08, \mathrm{CHCl}_{3}\right) ;{ }^{1} \mathrm{H}$ NMR $\left(400 \mathrm{MHz}, \mathrm{CDCl}_{3}\right) \delta 7.31(\mathrm{~d}, J=8.4 \mathrm{~Hz}, 2 \mathrm{H}), 7.18(\mathrm{~d}, J=8.4 \mathrm{~Hz}$, 2H), $4.91(\mathrm{dd}, J=4.8 \mathrm{~Hz}, 12.8 \mathrm{~Hz}, 1 \mathrm{H}), 4.85$ (dd, $J=8.4 \mathrm{~Hz}, 13.6 \mathrm{~Hz}, 1 \mathrm{H}), 4.23$ (dt, $J=$ $4.8 \mathrm{~Hz}, 9.2 \mathrm{~Hz}, 1 \mathrm{H}), 8.83(\mathrm{~d}, J=9.2 \mathrm{~Hz}, 1 \mathrm{H}), 3.77(\mathrm{~s}, 3 \mathrm{H}), 3.60(\mathrm{~s}, 3 \mathrm{H}) ;{ }^{13} \mathrm{C} \mathrm{NMR}(100$ $\left.\mathrm{MHz}_{\mathrm{CDCl}}\right) \delta 167.6,167.0,134.6,134.4,129.3,129.2,77.1,54.4,53.0,52.9,42.3$; IR $\left(\mathrm{CHCl}_{3}\right) \vee 3011,2956,1736,1653,1557,1494,1436,1259,1015 \mathrm{~cm}^{-1} ; \mathrm{HRMS}(\mathrm{CI}) \mathrm{m} / \mathrm{z}$ calcd. for $\left(\mathrm{C}_{13} \mathrm{H}_{14} \mathrm{ClNO}_{6}+\mathrm{H}^{+}\right)$: 316.0583 , found 316.0582 .

(-)-Methyl 2-carbomethoxy-4-nitro-3-(4-chloro-phenyl)butyrate (-)-6c This product was obtained as a white solid in $97 \%$ yield and $94 \%$ ee from a reaction catalyzed by QD-4a $(10 \mathrm{~mol} \%)$ at $-20^{\circ} \mathrm{C}$ for 36 hours.

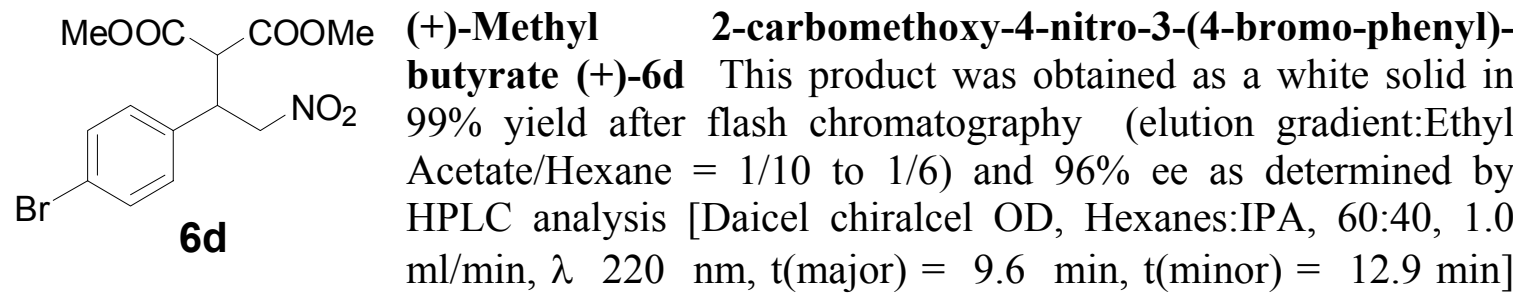
from a reaction catalyzed by $\mathbf{Q}-4 \mathbf{a}(10 \mathrm{~mol} \%)$ at $-20{ }^{\circ} \mathrm{C}$ for 36 hours. $[\alpha]_{\mathrm{D}}{ }^{25}=+6.9(\mathrm{c}$ 1.12, $\left.\mathrm{CHCl}_{3}\right) ;{ }^{1} \mathrm{H} \mathrm{NMR}\left(400 \mathrm{MHz}, \mathrm{CDCl}_{3}\right) \delta 7.46(\mathrm{~d}, J=8.4 \mathrm{~Hz}, 1 \mathrm{H}), 7.12(\mathrm{~d}, J=8.8$ $\mathrm{Hz}, 1 \mathrm{H}), 4.91(\mathrm{dd}, J=5.6 \mathrm{~Hz}, 13.6 \mathrm{~Hz}, 1 \mathrm{H}), 4.85(\mathrm{dd}, J=9.2 \mathrm{~Hz}, 13.6 \mathrm{~Hz}, 1 \mathrm{H}), 4.22 \mathrm{dt}, J$ $=4.8 \mathrm{~Hz}, 9.2 \mathrm{~Hz}, 1 \mathrm{H}), 3.82(\mathrm{~d}, J=9.2 \mathrm{~Hz}, 1 \mathrm{H}), 3.77(\mathrm{~s}, 3 \mathrm{H}), 3.60(\mathrm{~s}, 3 \mathrm{H}) ;{ }^{13} \mathrm{C} \mathrm{NMR}$ $\left(100 \mathrm{MHz}, \mathrm{CDCl}_{3}\right) \delta 167.6,167.0,135.1,132.2,129.6,122.5,77.1,54.4,53.1,52.9,42.3$; IR $\left(\mathrm{CHCl}_{3}\right) \vee 2952,1734,1653,1558,1507,1436 \mathrm{~cm}^{-1}$; HRMS (CI) m/z calcd. for $\left(\mathrm{C}_{13} \mathrm{H}_{14} \mathrm{BrNO}_{6}+\mathrm{H}^{+}\right): 360.0083$, found 360.0082 .

(-)-Methyl 2-carbomethoxy-4-nitro-3-(4-bromo-phenyl)-butyrate (-)-6d This product was obtained as a white solid in $98 \%$ yield and $94 \%$ ee from a reaction catalyzed by QD$4 \mathbf{a}(10 \mathrm{~mol} \%)$ at $-20{ }^{\circ} \mathrm{C}$ for 36 hours.

(+)-Methyl 2-carbomethoxy-4-nitro-3-(4-methylphenyl) butyrate $(+)-6 e$ This product was obtained as a colorless oil in $97 \%$ yield after flash chromatography (elution gradient: Ethyl Acetate/Hexane $=1 / 15$ to $1 / 10$ ) and $98 \%$ ee as determined by HPLC analysis 
MeOOC COOMe [Daicel chiralcel OD, Hexanes:IPA, 70:30, $1.0 \mathrm{ml} / \mathrm{min}, \lambda 220 \mathrm{~nm}$, $\mathrm{t}($ major $)=9.8 \mathrm{~min}, \mathrm{t}($ minor $)=11.0 \mathrm{~min}]$ from a reaction $\mathrm{NO}_{2}$ catalyzed by $\mathbf{Q}-4 \mathrm{a}(10 \mathrm{~mol} \%)$ at $-20^{\circ} \mathrm{C}$ for 36 hours. $[\alpha]_{\mathrm{D}}{ }^{25}=$ $+3.0\left(\mathrm{c} 1.40, \mathrm{CHCl}_{3}\right) ;{ }^{1} \mathrm{H} \mathrm{NMR}\left(400 \mathrm{MHz}, \mathrm{CDCl}_{3}\right) \delta$ 7.14-7.09 6e $\quad(\mathrm{m}, 4 \mathrm{H}), 4.93-4.82(\mathrm{~m}, 2 \mathrm{H}), 4.20(\mathrm{dt}, J=9.2 \mathrm{~Hz}, 5.6 \mathrm{~Hz}, 1 \mathrm{H})$, $3.85(\mathrm{~d}, J=9.2 \mathrm{~Hz}, 1 \mathrm{H}), 3.76(\mathrm{~s}, 3 \mathrm{H}), 3.58(\mathrm{~s}, 3 \mathrm{H}), 2.30(\mathrm{~s}, 3 \mathrm{H})$;

${ }^{13} \mathrm{C}$ NMR $\left(100 \mathrm{MHz}, \mathrm{CDCl}_{3}\right) \delta 167.9,167.2,138.1,132.9,129.7,127.6,77.5,54.7,52.9$, 52.8, 42.5, 21.1; IR $\left(\mathrm{CHCl}_{3}\right) \vee 3018,2956,1736,1557,1436,1259 \mathrm{~cm}^{-1} ; \mathrm{HRMS}(\mathrm{CI}) \mathrm{m} / \mathrm{z}$ calcd. for $\left(\mathrm{C}_{14} \mathrm{H}_{17} \mathrm{NO}_{6}+\mathrm{H}^{+}\right): 296.1134$, found 296.1141 .

(-)-Methyl 2-carbomethoxy-4-nitro-3-(4-methylphenyl)-butyrate (-)-6e This product was obtained as a colorless oil in $97 \%$ yield and $94 \%$ ee from a reaction catalyzed by QD-4a $(10 \mathrm{~mol} \%)$ at $-20{ }^{\circ} \mathrm{C}$ for 44 hours.

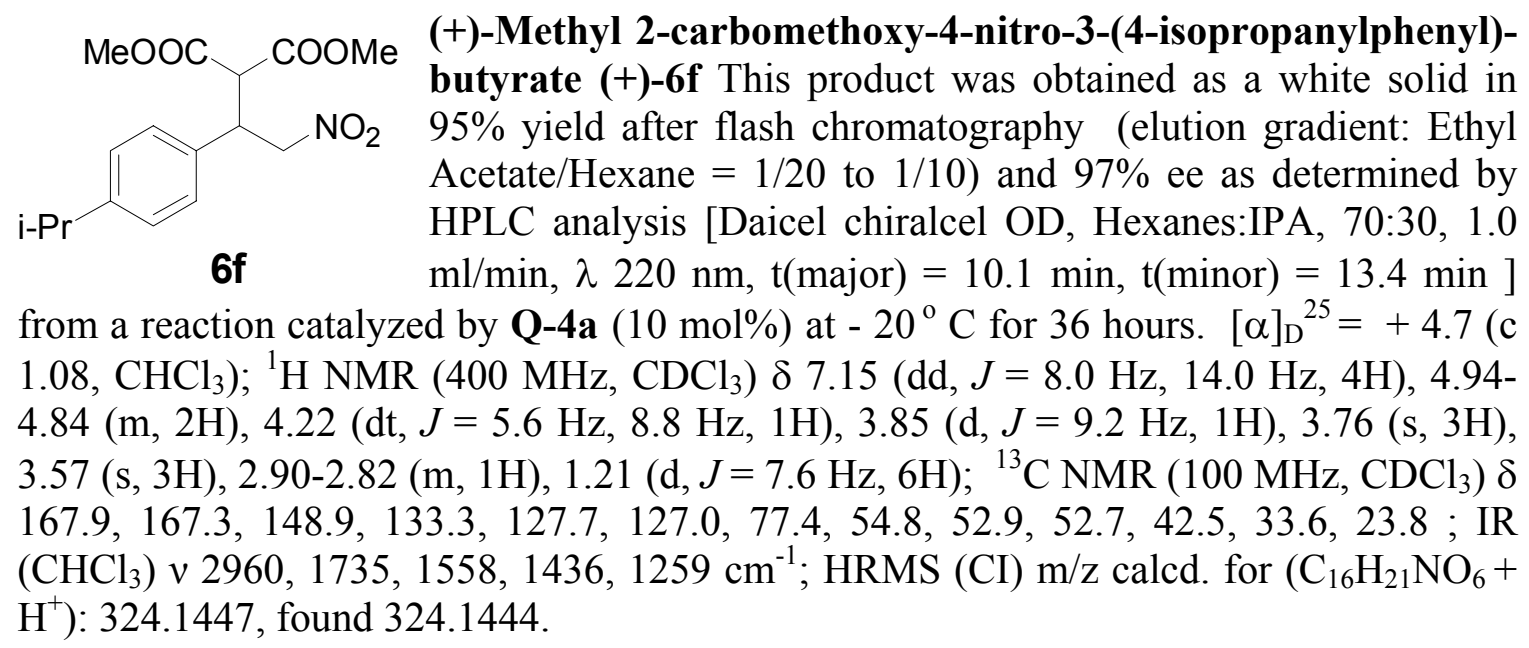

(-)-Methyl 2-carbomethoxy-4-nitro-3-(4-isopropanylphenyl)-butyrate (-)-6f This product was obtained as a a white solid in $96 \%$ yield and $93 \%$ ee from a reaction catalyzed by QD-4a $(10 \mathrm{~mol} \%)$ at $-20{ }^{\circ} \mathrm{C}$ for 39 hours.

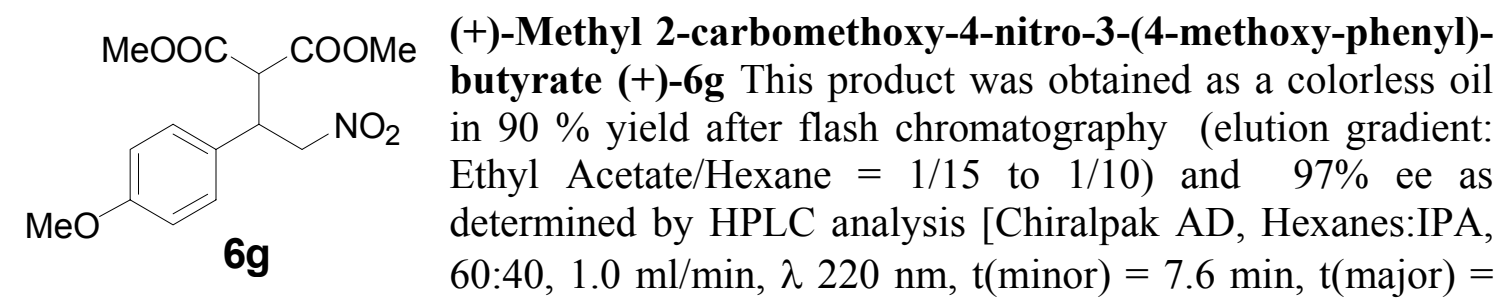
$11.9 \mathrm{~min}$ ] from a reaction catalyzed by $\mathbf{Q}-\mathbf{4 a}(10 \mathrm{~mol} \%)$ at $-20^{\circ} \mathrm{C}$ for 44 hours. $[\alpha]_{\mathrm{D}}{ }^{25}=$ $+5.0\left(\mathrm{c} 1.18, \mathrm{CHCl}_{3}\right) ;{ }^{1} \mathrm{H}$ NMR $\left(400 \mathrm{MHz}, \mathrm{CDCl}_{3}\right) \delta 7.14(\mathrm{~d}, J=9.2 \mathrm{~Hz}, 2 \mathrm{H}), 6.84(\mathrm{~d}, J$ $=8.8 \mathrm{~Hz}, 2 \mathrm{H}), 4.89(\mathrm{dd}, J=4.8 \mathrm{~Hz}, 12.8 \mathrm{~Hz}, 1 \mathrm{H}), 4.83(\mathrm{dd}, J=8.8 \mathrm{~Hz}, 13.6 \mathrm{~Hz}, 1 \mathrm{H})$, 4.19( dt, $J=4.8 \mathrm{~Hz}, 13.2 \mathrm{~Hz}, 1 \mathrm{H}), 3.82$ (d, $J=9.2 \mathrm{~Hz}, 1 \mathrm{H}), 3.78(\mathrm{~s}, 3 \mathrm{H}), 3.76(\mathrm{~s}, 3 \mathrm{H})$, $3.58(\mathrm{~s}, 3 \mathrm{H}) ;{ }^{13} \mathrm{C} \mathrm{NMR}\left(100 \mathrm{MHz}, \mathrm{CDCl}_{3}\right) \delta 167.9,167.3,159.4,129.0,127.8,114.3$, 77.6, 55.2, 54.8, 52.9, 52.8, 42.2; IR $\left(\mathrm{CHCl}_{3}\right) \vee$ 3011, 2957, 2841, 1733, 1557, 1516, 1436, 1258, $1032 \mathrm{~cm}^{-1}$; HRMS (CI) m/z calcd. for $\left(\mathrm{C}_{14} \mathrm{H}_{17} \mathrm{NO}_{7}+\mathrm{H}^{+}\right): 312.1070$, found 312.1076 . 
(-)-Methyl 2-carbomethoxy-4-nitro-3-(4-methoxy-phenyl)butyrate (-)-6g This product was obtained as a colorless oil in $94 \%$ yield and $95 \%$ ee from a reaction catalyzed by QD-4a (10 mol\%) at $-20^{\circ} \mathrm{C}$ for 47 hours.

\begin{tabular}{|c|c|c|}
\hline \multirow{4}{*}{\multicolumn{2}{|c|}{ MeOOC COOMe }} & (+)-Methyl 2-carbomethoxy-4-nitro-3-(3-methylphenyl)- \\
\hline & & \\
\hline & & Acetate / Hexane $=1 / 15$ to $1 / 10$ ) and $97 \%$ ee as determined by \\
\hline & & $\begin{array}{l}\text { HPLC analysis [Daicel chiralcel OD, Hexanes : IPA, 92:8, } 0.9 \\
\mathrm{ml} / \mathrm{min}, \lambda 220 \mathrm{~nm}, \mathrm{t} \text { (major) }=19.8 \mathrm{~min}, \mathrm{t} \text { (minor) }=22.2 \mathrm{~min}\end{array}$ \\
\hline
\end{tabular}
from a reaction catalyzed by $\mathbf{Q}-4 \mathbf{a}(10 \mathrm{~mol} \%)$ at $-20^{\circ} \mathrm{C}$ for 36 hours. $[\alpha]_{\mathrm{D}}{ }^{25}=+1.0(\mathrm{c} 1.00$, $\left.\mathrm{CHCl}_{3}\right) ;{ }^{1} \mathrm{H} \mathrm{NMR}\left(400 \mathrm{MHz}, \mathrm{CDCl}_{3}\right) \delta 7.21(\mathrm{t}, J=7.2 \mathrm{~Hz}, 1 \mathrm{H}), 7.09(\mathrm{~d}, J=7.2 \mathrm{~Hz}, 1 \mathrm{H})$, $7.02(\mathrm{~s}, 1 \mathrm{H}), 7.01(\mathrm{~d}, J=7.2 \mathrm{~Hz}, 1 \mathrm{H}), 4.94-4.84(\mathrm{~m}, 2 \mathrm{H}), 4.24-4.18(\mathrm{dt}, J=5.6 \mathrm{~Hz}, 8.4$ $\mathrm{Hz}, 1 \mathrm{H}), 3.86-3.84(\mathrm{~d}, J=8.4 \mathrm{~Hz}, 1 \mathrm{H}), 3.76(\mathrm{~s}, 3 \mathrm{H}), 3.58(\mathrm{~s}, 3 \mathrm{H}), 2.32(\mathrm{~s}, 3 \mathrm{H}) ;{ }^{13} \mathrm{C} \mathrm{NMR}$ $\left(100 \mathrm{MHz}, \mathrm{CDCl}_{3}\right) \delta 167.9,167.2,138.6,136.0,129.1,128.8,128.6,124.6,77.3,54.7$, 52.9, 52.8, 42.8, 21.3; IR $\left(\mathrm{CHCl}_{3}\right) \vee 3029,2957,1736,1557,1436,1259 \mathrm{~cm}^{-1}$; HRMS (CI) $\mathrm{m} / \mathrm{z}$ calcd. for $\left(\mathrm{C}_{14} \mathrm{H}_{17} \mathrm{NO}_{6}+\mathrm{H}^{+}\right): 296.1134$, found 296.1141 .

(-)-Methyl 2-carbomethoxy-4-nitro-3-(3-methylphenyl)butyrate (-)-6h This product was obtained as a colorless oil in $99 \%$ yield and $93 \%$ ee from a reaction catalyzed by QD-4a $(10 \mathrm{~mol} \%)$ at $-20{ }^{\circ} \mathrm{C}$ for 36 hours.

MeOOC COOMe (-)-Methyl 2-carbomethoxy-4-nitro-3-(2-methylphenyl)-butyrate ()-6i This product was obtained as a colorless oil in $95 \%$ yield after $\mathrm{NO}_{2}$ flash chromatography (elution gradient: Ethyl Acetate/Hexane $=1 / 15$ ) and $98 \%$ ee as determined by HPLC analysis [Chiralpak AD, Hexanes:IPA, 80:20, $1.0 \mathrm{ml} / \mathrm{min}, \lambda 220 \mathrm{~nm}, \mathrm{t}($ minor $)=6.5 \mathrm{~min}$, 6i $\quad t$ (major) $=12.2 \mathrm{~min}]$ from a reaction catalyzed by $\mathbf{Q}-4 \mathbf{a}(10 \mathrm{~mol} \%)$ at $-20{ }^{\circ} \mathrm{C}$ for 36 hours. $[\alpha]_{\mathrm{D}}{ }^{25}=-1.6\left(\mathrm{c} 1.25, \mathrm{CHCl}_{3}\right) ;{ }^{1} \mathrm{H}$ NMR $(400 \mathrm{MHz}$, $\left.\mathrm{CDCl}_{3}\right) \delta 7.17-7.11(\mathrm{~m}, 4 \mathrm{H}), 4.94-4.83(\mathrm{~m}, 2 \mathrm{H}), 4.57(\mathrm{dt}, J=5.6 \mathrm{~Hz}, 9.2 \mathrm{~Hz}, 1 \mathrm{H}), 3.83$ $(\mathrm{d}, J=9.2 \mathrm{~Hz}, 1 \mathrm{H}), 3.77(\mathrm{~s}, 3 \mathrm{H}), 3.54(\mathrm{~s}, 3 \mathrm{H}), 2.44(\mathrm{~s}, 3 \mathrm{H}) ;{ }^{13} \mathrm{C} \mathrm{NMR}\left(100 \mathrm{MHz}, \mathrm{CDCl}_{3}\right)$ $\delta 167.9,167.3,136.9,134.4,131.2,128.0,126.5,125.7,77.3,54.4,52.9,52.7,37.7,19.4$; IR $\left(\mathrm{CHCl}_{3}\right) \vee 3011,2956,1734,1558,1436,1260 \mathrm{~cm}^{-1}$; HRMS (CI) m/z calcd for $\left(\mathrm{C}_{14} \mathrm{H}_{17} \mathrm{NO}_{6}+\mathrm{H}^{+}\right): 296.1134$, found 296.1128 .

(+)-Methyl 2-carbomethoxy-4-nitro-3-(2-methylphenyl)butyrate (+)-6i This product was obtained as a colorless oil in $97 \%$ yield and $96 \%$ ee from a reaction catalyzed by QD-4a (10 mol\%) at $-20{ }^{\circ} \mathrm{C}$ for 36 hours.

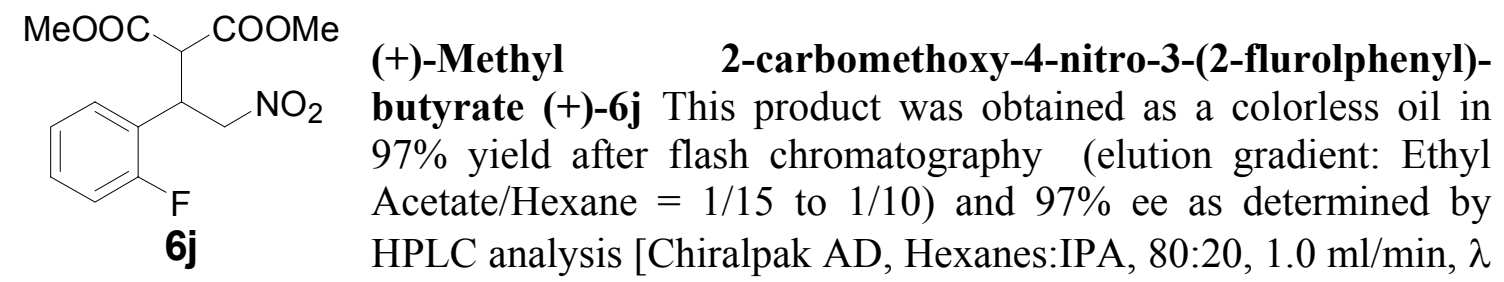


$220 \mathrm{~nm}, \mathrm{t}($ minor $)=8.2 \mathrm{~min}, \mathrm{t}$ (major) $=10.1 \mathrm{~min}]$ from a reaction catalyzed by $\mathbf{Q}-\mathbf{4 a}(10$ mol\%) at $-20^{\circ} \mathrm{C}$ for 36 hours. $[\alpha]_{\mathrm{D}}{ }^{25}=+14.6\left(\mathrm{c} 1.08, \mathrm{CHCl}_{3}\right) ;{ }^{1} \mathrm{H} \mathrm{NMR}(400 \mathrm{MHz}$, $\left.\mathrm{CDCl}_{3}\right) \delta$ 7.32-7.22 (m, 2H), 7.12-7.05 (m, 2H), 4.95-4.93 (d, $\left.J=7.2 \mathrm{~Hz}, 2 \mathrm{H}\right), 4.46-4.4$ $(\mathrm{m}, 1 \mathrm{H}), 4.00(\mathrm{~d}, J=9.6 \mathrm{~Hz}, 1 \mathrm{H}), 3.78(\mathrm{~s}, 3 \mathrm{H}), 3.56(\mathrm{~s}, 3 \mathrm{H}) ;{ }^{13} \mathrm{C} \mathrm{NMR}(100 \mathrm{MHz}$, $\left.\mathrm{CDCl}_{3}\right) \delta 167.7,167.1,162.1,159.7,130.47,130.44,130.36,130.3,124.61,24.58,123.0$, 122.9, 116.2, 116.0, 76.00, 75.98, 53.05, 52.99, 52.97, 52.81, 38.5; IR $\left(\mathrm{CHCl}_{3}\right) \vee 3011$, $2958,2848,1733,1557,1495,1436,1259 \mathrm{~cm}^{-1}$; HRMS (CI) m/z calcd. for $\left(\mathrm{C}_{13} \mathrm{H}_{14} \mathrm{FNO}_{6}\right.$ $+\mathrm{H}^{+}$): 300.0883 , found 300.0879 .

(-)-Methyl 2-carbomethoxy-4-nitro-3-(2-flurolphenyl)butyrate (-)-6j This product was obtained as a colorless oil in $94 \%$ yield and $95 \%$ ee from a reaction catalyzed by QD-4a (10 mol\%) at $-20^{\circ} \mathrm{C}$ for 36 hours.

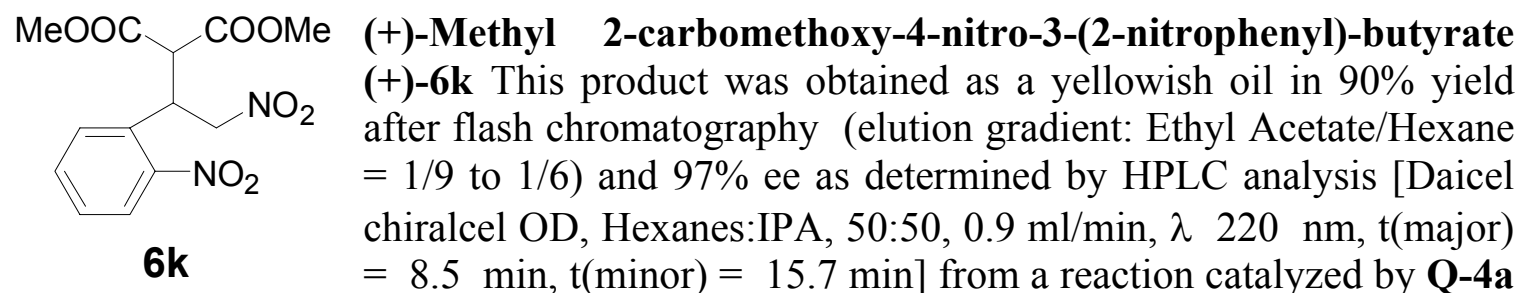
$(10 \mathrm{~mol} \%)$ at $-55^{\circ} \mathrm{C}$ for 69 hours. $[\alpha]_{\mathrm{D}}{ }^{25}=+18.9\left(\mathrm{c} 1.04, \mathrm{CHCl}_{3}\right) ;{ }^{1} \mathrm{H}$ NMR $(400 \mathrm{MHz}$, $\left.\mathrm{CDCl}_{3}\right) \delta 7.95(\mathrm{dd}, J=1.2 \mathrm{~Hz}, 8.8 \mathrm{~Hz}, 1 \mathrm{H}), 7.60(\mathrm{dt}, J=1.2 \mathrm{~Hz}, 8.0 \mathrm{~Hz}, 1 \mathrm{H}), 7.49(\mathrm{dt}, J$ $=1.2 \mathrm{~Hz}, 8.0 \mathrm{~Hz}, 1 \mathrm{H}), 7.41(\mathrm{dd}, J=1.2 \mathrm{~Hz}, 8.0 \mathrm{~Hz}, 1 \mathrm{H}), 5.17(\mathrm{dd}, J=8.0 \mathrm{~Hz}, 14.0 \mathrm{~Hz}$, $1 \mathrm{H}), 5.06(\mathrm{dd}, J=4.0 \mathrm{~Hz}, 14.0 \mathrm{~Hz}, 1 \mathrm{H}), 4.77(\mathrm{td}, J=4.0 \mathrm{~Hz}, 8.0 \mathrm{~Hz}, 1 \mathrm{H}), 4.25(\mathrm{~d}, J=8.0$ $\mathrm{Hz}, 1 \mathrm{H}), 3.77$ (s, 3H), 3.65 (s, 3H); ${ }^{13} \mathrm{C}$ NMR $\left(100 \mathrm{MHz}, \mathrm{CDCl}_{3}\right) \delta 167.6,167.0,149.8$, 133.3, 131.1, 129.3, 128.7, 125.4, 75.9, 53.4, 53.11, 53.08, 37.4; IR $\left(\mathrm{CHCl}_{3}\right) \vee 3018$, 2958, 1733, 1558, 1436, $1361 \mathrm{~cm}^{-1}$; HRMS (CI) m/z calcd. for $\left(\mathrm{C}_{13} \mathrm{H}_{14} \mathrm{~N}_{2} \mathrm{O}_{8}+\mathrm{H}^{+}\right)$: 327.0828 , found 327.0822 .

(-)-Methyl 2-carbomethoxy-4-nitro-3-(2-nitrophenyl)butyrate (-)-6k This product was obtained as a yellowish oil in $88 \%$ yield and $92 \%$ ee from a reaction catalyzed by QD-4a $(10 \mathrm{~mol} \%)$ at $-55^{\circ} \mathrm{C}$ for 72 hours.

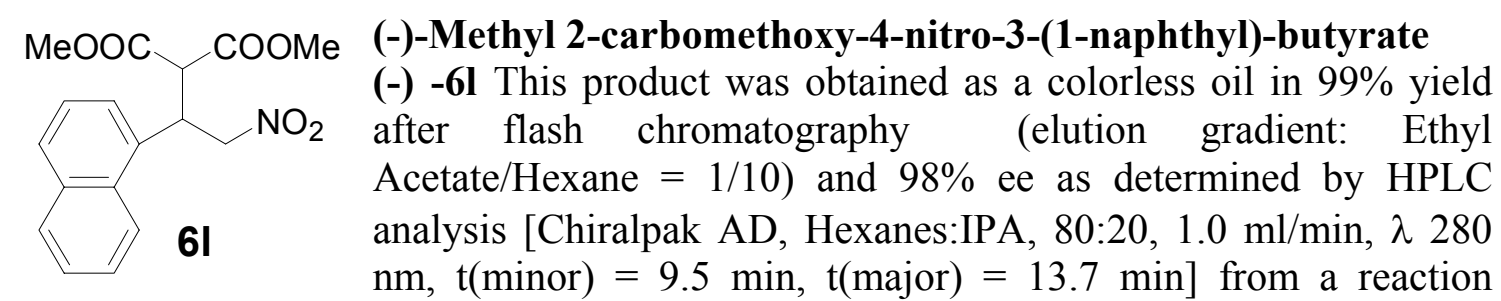
catalyzed by Q-4a $(10 \mathrm{~mol} \%)$ at $-20^{\circ} \mathrm{C}$ for 36 hours. $[\alpha]_{\mathrm{D}}{ }^{25}=-3.8\left(\mathrm{c} 1.32, \mathrm{CHCl}_{3}\right) ;{ }^{1} \mathrm{H}$ NMR $\left(400 \mathrm{MHz}, \mathrm{CDCl}_{3}\right) \delta 8.18(\mathrm{~d}, J=8.4 \mathrm{~Hz}, 1 \mathrm{H}), 7.88(\mathrm{~d}, J=8.8 \mathrm{~Hz}, 1 \mathrm{H}), 7.80(\mathrm{~d}, J=$ $8.0 \mathrm{~Hz}, 1 \mathrm{H}), 7.62$ (t, $J=8.0 \mathrm{~Hz}, 1 \mathrm{H}), 7.53$ (t, $J=8.0 \mathrm{~Hz}, 1 \mathrm{H}), 7.45-7.28(\mathrm{~m}, 2 \mathrm{H}), 5.24-$ $5.05(\mathrm{~m}, 3 \mathrm{H}), 4.13-4.10(\mathrm{~d}, J=7.2 \mathrm{~Hz}, 1 \mathrm{H}), 3.72(\mathrm{~s}, 3 \mathrm{H}), 3.55(\mathrm{~s}, 3 \mathrm{H}) ;{ }^{13} \mathrm{C}$ NMR $(100$ $\left.\mathrm{MHz}, \mathrm{CDCl}_{3}\right) \delta 167.9,167.4,134.1,132.2,130.9,129.2,128.9,127.0,126.1,125.1$, 123.9, 122.1, 76.6, 54.3, 52.9, 36.7; IR $\left(\mathrm{CHCl}_{3}\right)$ v 3040, 3011, 2955, 1734, 1557, 1436, 1252, $1028 \mathrm{~cm}^{-1}$; HRMS (CI) $\mathrm{m} / \mathrm{z}$ calcd. for $\left(\mathrm{C}_{17} \mathrm{H}_{17} \mathrm{NO}_{6}+\mathrm{H}^{+}\right): 311.1056$, found 311.1055 . 
(+)-Methyl 2-carbomethoxy-4-nitro-3-(1-naphthyl)butyrate $(+)-61$ This product was obtained as a colorless oil in $99 \%$ yield and $92 \%$ ee from a reaction catalyzed by QD-4a $(10 \mathrm{~mol} \%)$ at $-20{ }^{\circ} \mathrm{C}$ for 36 hours.

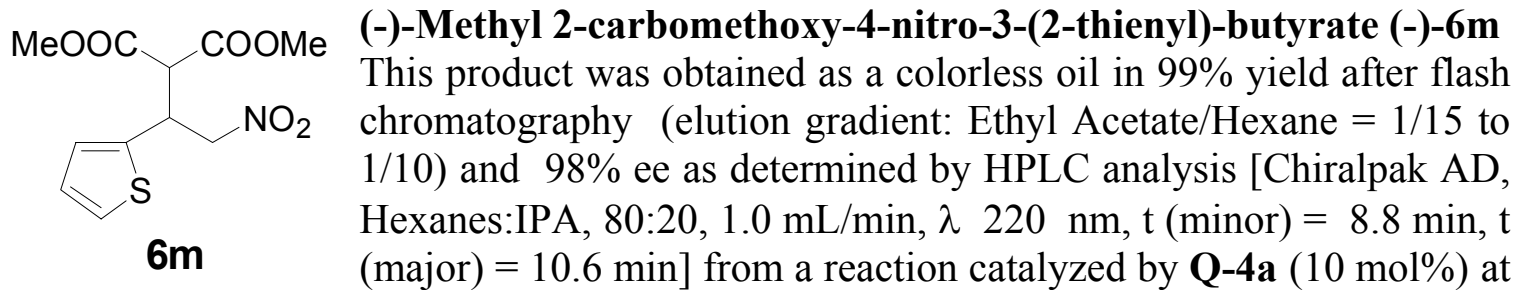
$-20^{\circ} \mathrm{C}$ for 36 hours. $[\alpha]_{\mathrm{D}}{ }^{25}=-7.8\left(\mathrm{c} 2.70, \mathrm{CHCl}_{3}\right) ;{ }^{1} \mathrm{H} \mathrm{NMR}\left(400 \mathrm{MHz}, \mathrm{CDCl}_{3}\right) \delta 7.24$ $(\mathrm{d}, J=5.2 \mathrm{~Hz}, 1 \mathrm{H}), 6.96-6.93(\mathrm{~m}, 2 \mathrm{H}), 4.98-4.89(\mathrm{~m}, 2 \mathrm{H}), 4.60-4.54(\mathrm{dt}, J=5.2 \mathrm{~Hz}$, $8.0 \mathrm{~Hz}, 1 \mathrm{H}), 3.92(\mathrm{~d}, J=7.2 \mathrm{~Hz}, 1 \mathrm{H}), 3.77(\mathrm{~s}, 3 \mathrm{H}), 3.68(\mathrm{~s}, 3 \mathrm{H}) ;{ }^{13} \mathrm{C}$ NMR $(100 \mathrm{MHz}$, $\left.\mathrm{CDCl}_{3}\right) \delta 167.6,167.1,138.3,127.1,126.7,125.6,77.8,55.2,53.1,53.0,38.3 ; \mathrm{IR}\left(\mathrm{CHCl}_{3}\right)$ $v$ 3099, 3003, 2957, 1733, 1557, 1436, $1264 \mathrm{~cm}^{-1}$; HRMS (CI) m/z calcd. for $\left(\mathrm{C}_{11} \mathrm{H}_{13} \mathrm{NO}_{6} \mathrm{~S}+\mathrm{H}^{+}\right): 288.0528$, found 288.0530 .

(+)-Methyl 2-carbomethoxy-4-nitro-3-(2-thienyl)butyrate $(+)-6 \mathrm{~m}$ This product was obtained as a colorless oil in $96 \%$ yield and $95 \%$ ee from a reaction catalyzed by QD-4a (10 mol\%) at $-20{ }^{\circ} \mathrm{C}$ for 44 hours.

MeOOC COOMe (-)-Methyl 2-carbomethoxy-4-nitro-3-(2-furyl)-butyrate (-)-6n This product was obtained as a yellowish oil in $97 \%$ yield after $\mathrm{NO}_{2}$ flash chromatography (elution grdient: Ethyl Acetate / Hexane = $1 / 15$ ) and $98 \%$ ee as determined by HPLC analysis [Daicel $6 n \quad$ chiralcel OD, Hexanes:IPA, 60:40, $1.0 \mathrm{ml} / \mathrm{min}, \lambda 220 \mathrm{~nm}, \mathrm{t}$ (major) $=6.2 \mathrm{~min}, \mathrm{t}($ minor $)=14.9 \mathrm{~min}]$ from a reaction catalyzed by $\mathbf{Q}-4 \mathbf{a}(10 \mathrm{~mol} \%)$ at $-20^{\circ}$ C for 36 hours. $[\alpha]_{\mathrm{D}}{ }^{25}=-4.9\left(\mathrm{c} 1.10, \mathrm{CHCl}_{3}\right) ;{ }^{1} \mathrm{H} \mathrm{NMR}\left(400 \mathrm{MHz}, \mathrm{CDCl}_{3}\right) \delta 7.35(\mathrm{~d}, J=$ $1.6 \mathrm{~Hz}, 1 \mathrm{H}), 6.30(\mathrm{dd}, J=1.6 \mathrm{~Hz}, 3.2 \mathrm{~Hz}, 1 \mathrm{H}), 6.23(\mathrm{~d}, J=3.2 \mathrm{~Hz}, 1 \mathrm{H}), 4.96-4.86(\mathrm{~m}$, 2H), 4.39 (dt, $J=4.8 \mathrm{~Hz}, 8.0 \mathrm{~Hz}, 1 \mathrm{H}), 3.95$ (d, $J=8.0 \mathrm{~Hz}, 1 \mathrm{H}), 3.77(\mathrm{~s}, 3 \mathrm{H}), 3.70(\mathrm{~s}, 3 \mathrm{H})$; ${ }^{13} \mathrm{C}$ NMR $\left(100 \mathrm{MHz}, \mathrm{CDCl}_{3}\right) \delta 167.5,167.2,149.3,142.8,110.5,108.4,75.2,53.03$, 52.99, 52.5, 36.7; IR $\left(\mathrm{CHCl}_{3}\right) \vee$ 3018, 2958, 1733, 1558, 1436, 1263, $1014 \mathrm{~cm}^{-1}$; HRMS (CI) $\mathrm{m} / \mathrm{z}$ calcd. for $\left(\mathrm{C}_{11} \mathrm{H}_{13} \mathrm{NO}_{7}+\mathrm{H}^{+}\right)$: 272.0770, found 272.0768 .

(+)-Methyl 2-carbomethoxy-4-nitro-3-(2-furyl)-butyrate (+)-6n This product was obtained as a yellowish oil in $99 \%$ yield and $96 \%$ ee from a reaction catalyzed by QD-4a (10 mol\%) at $-20^{\circ} \mathrm{C}$ for 36 hours.

MeOOC COOMe (+)-Methyl 2-carbomethoxy-4-nitro-3-(3-pyridinyl)-butyrate (+)-

$\mathrm{NO}_{2} \quad 60$ This product was obtained as a white solid in $98 \%$ yield after flash chromatography (elution grdient: Ethyl Acetate/Hexane $=1 / 2$

to $1 / 1$ ) and $96 \%$ ee as determined by HPLC analysis [Chiralpak AD, $60 \quad H e x a n e s: I P A, 60: 40,1.0 \mathrm{ml} / \mathrm{min}, \lambda 220 \mathrm{~nm}, \mathrm{t}($ minor $)=6.6 \mathrm{~min}$, $\mathrm{t}($ major $)=9.2 \mathrm{~min}]$ from a reaction catalyzed by $\mathbf{Q}-4 \mathbf{a}(10 \mathrm{~mol} \%)$ at $-20^{\circ} \mathrm{C}$ for 36 hours. $[\alpha]_{\mathrm{D}}{ }^{25}=+3.3\left(\mathrm{c} 1.30, \mathrm{CHCl}_{3}\right) ;{ }^{1} \mathrm{H} \mathrm{NMR}\left(400 \mathrm{MHz}, \mathrm{CDCl}_{3}\right) \delta: 8.56(\mathrm{dd}, J=1.6 \mathrm{~Hz}, 4.8$ 
$\mathrm{Hz}, 1 \mathrm{H}), 8.54(\mathrm{~d}, J=2.8 \mathrm{~Hz}, 1 \mathrm{H}), 7.61(\mathrm{dt}, J=8.0 \mathrm{~Hz}, 2.0 \mathrm{~Hz}, 1 \mathrm{H}), 7.28(\mathrm{dd}, J=4.8 \mathrm{~Hz}$, $8.0 \mathrm{~Hz}, 1 \mathrm{H}), 4.99-4.88(\mathrm{~m}, 2 \mathrm{H}), 4.28(\mathrm{dt}, J=5.2 \mathrm{~Hz}, 9.2 \mathrm{~Hz}, 1 \mathrm{H}), 3.88(\mathrm{~d}, J=9.2 \mathrm{~Hz}$, $1 \mathrm{H}), 3.78(\mathrm{~s}, 3 \mathrm{H}), 3.61$ (s, 3H); ${ }^{13} \mathrm{C} \mathrm{NMR}\left(100 \mathrm{MHz}, \mathrm{CDCl}_{3}\right) \delta 167.4,166.9,149.8,149.6$, 135.3, 131.9, 123.6, 76.7, 54.1, 53.2, 53.0, 40.5; IR $\left(\mathrm{CHCl}_{3}\right)$ v 3025, 2957, 1733, 1558, 1435, 1265, $1025 \mathrm{~cm}^{-1}$; HRMS (CI) m/z calcd. for $\left(\mathrm{C}_{12} \mathrm{H}_{14} \mathrm{~N}_{2} \mathrm{O}_{6}+\mathrm{H}^{+}\right): 283.0930$, found 283.0923 .

(-)-Methyl 2-carbomethoxy-4-nitro-3-(3-pyridinyl)butyrate (-)-6o This product was obtained as a white solid in $99 \%$ yield and $92 \%$ ee from a reaction catalyzed by QD-4a $(10 \mathrm{~mol} \%)$ at $-20{ }^{\circ} \mathrm{C}$ for 36 hours.

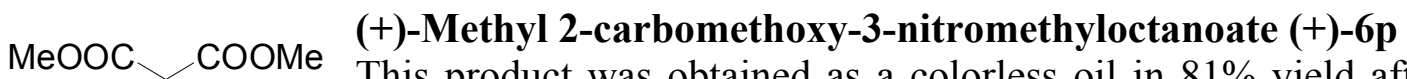

This product was obtained as a colorless oil in $81 \%$ yield after $\mathrm{NO}_{2}$ flash chromatography (elution gradient: Ethyl Acetate/Hexane = $1 / 20$ ) and $94 \%$ ee as determined by HPLC analysis [Daicel $6 p \quad$ chiralcel OD, Hexanes:IPA, 90:10, $1.0 \mathrm{ml} / \mathrm{min}, \lambda 215 \mathrm{~nm}, \mathrm{t}$ $($ major $)=6.1 \mathrm{~min}, \mathrm{t}($ minor $)=12.3 \mathrm{~min}]$ from a reaction catalyzed by $\mathbf{Q}-4 \mathbf{a}(10 \mathrm{~mol} \%)$ at $-20{ }^{\circ} \mathrm{C}$ for 72 hours. $[\alpha]_{\mathrm{D}}{ }^{25}=+7.6\left(\mathrm{c} 1.75, \mathrm{CHCl}_{3}\right) ;{ }^{1} \mathrm{H} \mathrm{NMR}\left(400 \mathrm{MHz}, \mathrm{CDCl}_{3}\right) \delta 4.71$ (dd, $J=5.6 \mathrm{~Hz}, 13.2 \mathrm{~Hz}, 1 \mathrm{H}), 4.53(\mathrm{dd}, J=6.8 \mathrm{~Hz}, 13.6 \mathrm{~Hz}, 1 \mathrm{H}), 3.77$ (s, 3H), 3.76 (s, $3 \mathrm{H}), 3.68(\mathrm{~d}, J=5.6 \mathrm{~Hz}, 1 \mathrm{H}), 2.92-2.87(\mathrm{~m}, 1 \mathrm{H}), 1.48-1.42(\mathrm{~m}, 2 \mathrm{H}), 1.39-1.26(\mathrm{~m}, 6 \mathrm{H})$, $0.88(\mathrm{t}, J=6.4 \mathrm{~Hz}, 3 \mathrm{H}) ;{ }^{13} \mathrm{C} \mathrm{NMR}\left(100 \mathrm{MHz}, \mathrm{CDCl}_{3}\right) \delta 168.4,168.2,76.5,52.8,52.7$, 52.3, 36.9, 31.3, 29.9, 26.2, 22.3, 13.9; IR $\left(\mathrm{CHCl}_{3}\right) \vee 2957,2929,1735,1558,1436,1160$ $\mathrm{cm}^{-1}$; HRMS (CI) m/z calcd. for $\left(\mathrm{C}_{12} \mathrm{H}_{21} \mathrm{NO}_{6}+\mathrm{H}^{+}\right): 276.1447$, found 276.1446.

(-)-Methyl 2-carbomethoxy-3-nitromethyloctanoate (-)-6p This product was obtained as a colorless oil in $82 \%$ yield and $91 \%$ ee from a reaction catalyzed by QD-4a (10 mol\%) at $-20{ }^{\circ} \mathrm{C}$ for 72 hours.

MeOOC COOMe (+)-Methyl 2-carbomethoxy-5-methyl-3-nitromethylhexanoate $(+)-6 q$ This product was obtained as a colorless oil in $86 \%$ yield after flash chromatography (elution gradient: Ethyl Acetate/Hexane

$6 \mathbf{q}=1 / 20$ ) and $94 \%$ ee as determined by HPLC analysis [Daicel chiralcel OD, Hexanes:IPA, 90:10, $1.0 \mathrm{ml} / \mathrm{min}, \lambda 215 \mathrm{~nm}, \mathrm{t}$ (major) $=5.6 \mathrm{~min}, \mathrm{t}$ (minor) $=$ $11.2 \mathrm{~min}]$ from a reaction catalyzed by $\mathbf{Q}-\mathbf{4 a}(10 \mathrm{~mol} \%)$ at $-20^{\circ} \mathrm{C}$ for 72 hours. $[\alpha]_{\mathrm{D}}{ }^{25}$ $=+9.0\left(\mathrm{c} 2.05, \mathrm{CHCl}_{3}\right) ;{ }^{1} \mathrm{H} \mathrm{NMR}\left(400 \mathrm{MHz}, \mathrm{CDCl}_{3}\right) \delta 4.68(\mathrm{dd}, J=4.8 \mathrm{~Hz}, 13.6 \mathrm{~Hz}$, $1 \mathrm{H}), 4.49$ (dd, $J=6.4 \mathrm{~Hz}, 13.6 \mathrm{~Hz}, 1 \mathrm{H}), 3.740$ (s, 3H), $3.736(\mathrm{~s}, 3 \mathrm{H}), 3.63$ (d, $J=5.6 \mathrm{~Hz}$, $1 \mathrm{H}), 2.96-2.91(\mathrm{~m}, 1 \mathrm{H}), 1.65-1.58(\mathrm{~m}, 1 \mathrm{H}), 1.32-1.22(\mathrm{~m}, 2 \mathrm{H}), 0.90-0.88(\mathrm{~m}, 6 \mathrm{H}) ;{ }^{13} \mathrm{C}$ NMR $\left(100 \mathrm{MHz}, \mathrm{CDCl}_{3}\right) \delta 168.4,168.2,76.7,52.8,52.7,52.3,38.9,34.9,25.1,22.3$, 22.1; IR $\left(\mathrm{CHCl}_{3}\right) \vee 2959,2878,1732,1557,1436,1161,1027 \mathrm{~cm}^{-1} ; \mathrm{HRMS}(\mathrm{CI}) \mathrm{m} / \mathrm{z}$ calcd. for $\left(\mathrm{C}_{11} \mathrm{H}_{19} \mathrm{NO}_{6}+\mathrm{H}^{+}\right)$: 262.1291, found 262.1297.

(-)-Methyl 2-carbomethoxy-5-methyl-3-nitromethylhexanoate (-)-6q This product was obtained as a colorless oil in $84 \%$ yield and $92 \%$ ee from a reaction catalyzed by QD-4a $(10 \mathrm{~mol} \%)$ at $-20{ }^{\circ} \mathrm{C}$ for 72 hours. 


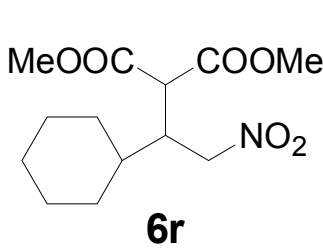

$(+)$-Methyl 2-carbomethoxy-4-nitro-3-cyclohexylbutyrate (+)6r

This product was obtained as a colorless oil in $71 \%$ yield after flash chromatography (elution gradient: Ethyl Acetate/Hexane = $1 / 20$ ) and $94 \%$ ee as determined by HPLC analysis [Daicel chiralcel OD, Hexanes:IPA, 90:10, $1.0 \mathrm{ml} / \mathrm{min}, \lambda 215 \mathrm{~nm}, \mathrm{t}$ (major) $=6.0 \mathrm{~min}, \mathrm{t}($ minor $)=14.8 \mathrm{~min}]$ from a reaction catalyzed by $\mathbf{Q}-\mathbf{4 a}(20 \mathrm{~mol} \%)$ at $-20^{\circ} \mathrm{C}$ for 108 hours. $[\alpha]_{\mathrm{D}}{ }^{25}=+12.0\left(\mathrm{c} 0.95, \mathrm{CHCl}_{3}\right) ;{ }^{1} \mathrm{H}$ NMR $\left(400 \mathrm{MHz}, \mathrm{CDCl}_{3}\right) \delta 4.73(\mathrm{dd}, J$ $=4.4 \mathrm{~Hz}, 14.8 \mathrm{~Hz}, 1 \mathrm{H}), 4.62(\mathrm{dd}, J=6.8 \mathrm{~Hz}, 14.8 \mathrm{~Hz}, 1 \mathrm{H}), 3.77-3.75(\mathrm{~m}, 7 \mathrm{H}), 2.92-2.88$ $(\mathrm{m}, 1 \mathrm{H}), 1.78-1.59(\mathrm{~m}, 5 \mathrm{H}), 1.49-1.42(\mathrm{~m}, 1 \mathrm{H}), 1.26-1.10(\mathrm{~m}, 3 \mathrm{H}), 1.07-0.95(\mathrm{~m}, 2 \mathrm{H}) ;{ }^{13} \mathrm{C}$ NMR $\left(100 \mathrm{MHz}, \mathrm{CDCl}_{3}\right) \delta 168.9,168.6,75.4,52.9,52.7,50.9,42.1,39.6,30.1,29.8$, 26.22, 26.17, 25.9; IR $\left(\mathrm{CHCl}_{3}\right) \vee 2931,2855,1735,1557,1436,1160,1024 \mathrm{~cm}^{-1}$; HRMS (CI) $\mathrm{m} / \mathrm{z}$ calcd. for $\left(\mathrm{C}_{13} \mathrm{H}_{21} \mathrm{NO}_{6}+\mathrm{H}^{+}\right): 288.1447$, found 288.1443 .

(-)-Methyl 2-carbomethoxy-4-nitro-3-cyclohexylbutyrate (-)-6r This product was obtained as a colorless oil in $80 \%$ yield and $91 \%$ ee from a reaction catalyzed by QD-4a (20 mol\%) at $-20^{\circ} \mathrm{C}$ for 108 hours.

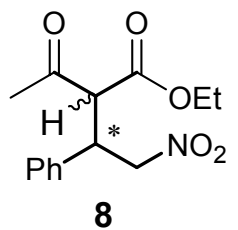

Ethyl 2-acetyl-4-3-phenylbutyrate 8 This product was obtained as a white solid in $93 \%$ yield after flash chromatography (elution gradient: Ethyl Acetate/Hexane $=1 / 10$ to $1 / 5$ )as a $\sim 1 / 1$ mixture of two compounds which are diastereomeric at the ester-bearing stereocenter (which agrees with the literature report ${ }^{4}$ ) and $91 \%$ ee as determined by HPLC analysis [Daicel chiralpak AD, Hexanes:ethanol, 90:10, $0.8 \mathrm{ml} / \mathrm{min}, \lambda 210 \mathrm{~nm}$, $\mathrm{t}$ (major) $=12.8$ $\mathrm{min}, 19.8 \mathrm{~min}, \mathrm{t}($ minor $)=17.3 \mathrm{~min}, 22.3 \mathrm{~min}]$ from a reaction catalyzed by $\mathbf{Q}-\mathbf{4 a}(10$ mol\%) at $23{ }^{\circ} \mathrm{C}$ for 6 hours.

\section{Kinetic Data:}

The kinetic parameters of this reaction were determined by in situ monitoring of the consumption of nitroalkenes (at peak $1522 \mathrm{~cm}^{-1}$ ) by the use of a ReactIR 1000 instrument. ReactIR 1000 fitted with a 5/8" Dicomp Probe, running software version 2.1a.

Order in nitroalkene (5a) was established by using a large excess of Dimethyl malonate (5 equiv) and $10 \mathrm{~mol} \%$ QD-4a. Plotting in $\ln [\mathbf{5 a}]$ versus time gives a straight line $\left(\mathrm{R}^{2}=\right.$ 0.9999, Figure A), thus establishing first-order dependence in nitroalkene $\mathbf{5 a}$.

Order in dimethyl malonate was established by using a large excess of nitroalkene (5a, 5 equiv) and $10 \mathrm{~mol} \%$ QD-4a. In this case, the reaction was monitored by following the formation of the product (at peak $1557 \mathrm{~cm}^{-1}$ ). Plotting in $\ln$ [Dimethyl malonate] versus time gives a straight line $\left(\mathrm{R}^{2}=0.9975\right.$, Figure $\left.\mathrm{C}\right)$, thus establishing the first-order dependence in malonate.

The reaction order in catalyst was established by determining the rate constants $\left(\mathrm{k}_{\mathrm{obs}}\right)$ at various catalyst concentrations. A plot of the rate constants $\mathrm{k}_{\mathrm{obs}}$ versus the catalyst 
loading gives a straight line for QD-4a $\left(\mathrm{R}^{2}=0.99\right.$, Figure $\left.\mathrm{F}\right)$, indicating the first-order dependence on catalyst.

General procedure for kinetic study:

A mixture of Nitroalkene (5a) $(1.0 \mathrm{mmol})$ and QD-4a $(5-15 \mathrm{~mol} \%)$ in anhydrous THF $(1.0 \mathrm{~mL})$ was stirred at room temperature for 5 minutes, and then dimethyl malonate $(0.57 \mathrm{ml}, 5 \mathrm{eq})$ was introduced in one portion via a syringe. The resulting reaction mixture was monitored every 2 minutes for 6 hours.

Figure A. Determination of the order of Nitroalkene (5a)

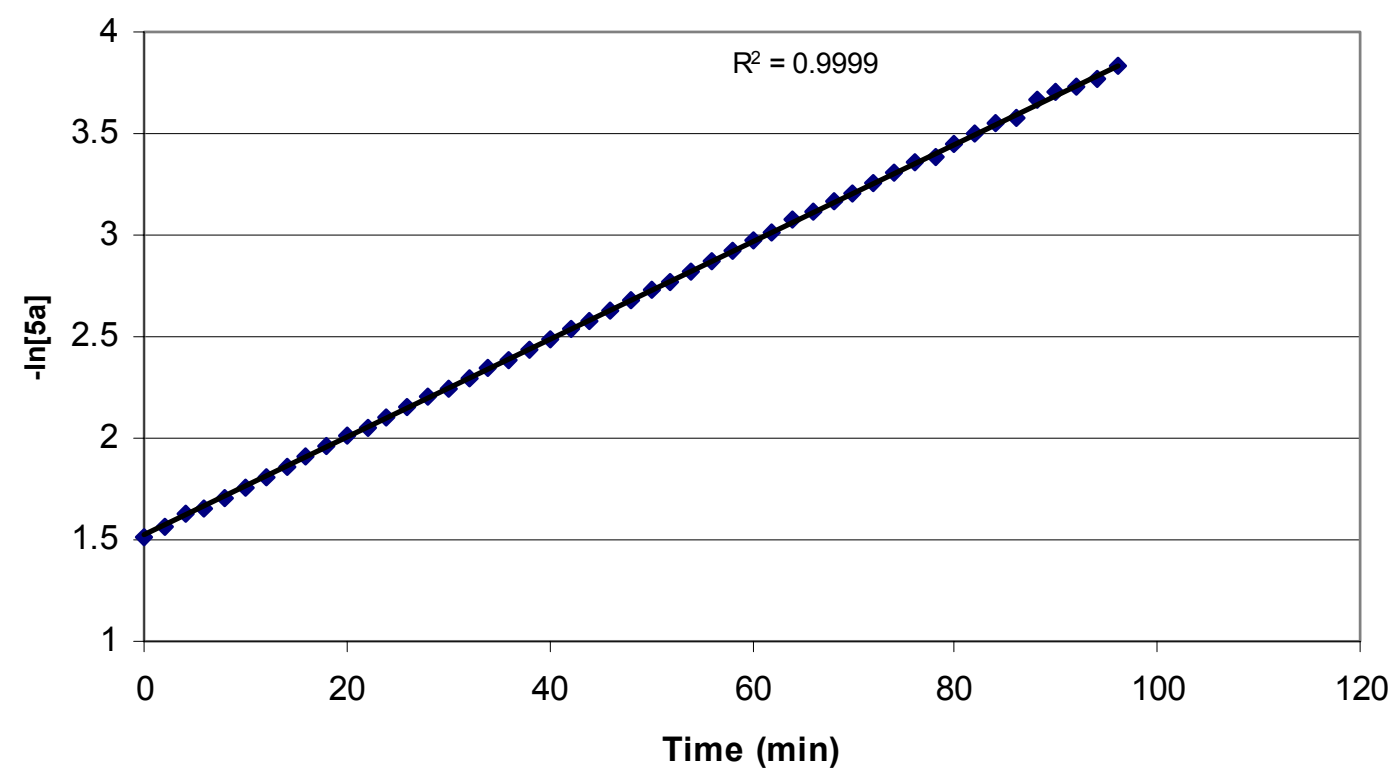

Figure A shows a linear relationship between $\ln [\mathbf{5 a}]$ and time, indicating the reaction is first order in 5a.

Figure B. Determination of the order of Nitroalkene (5a) 


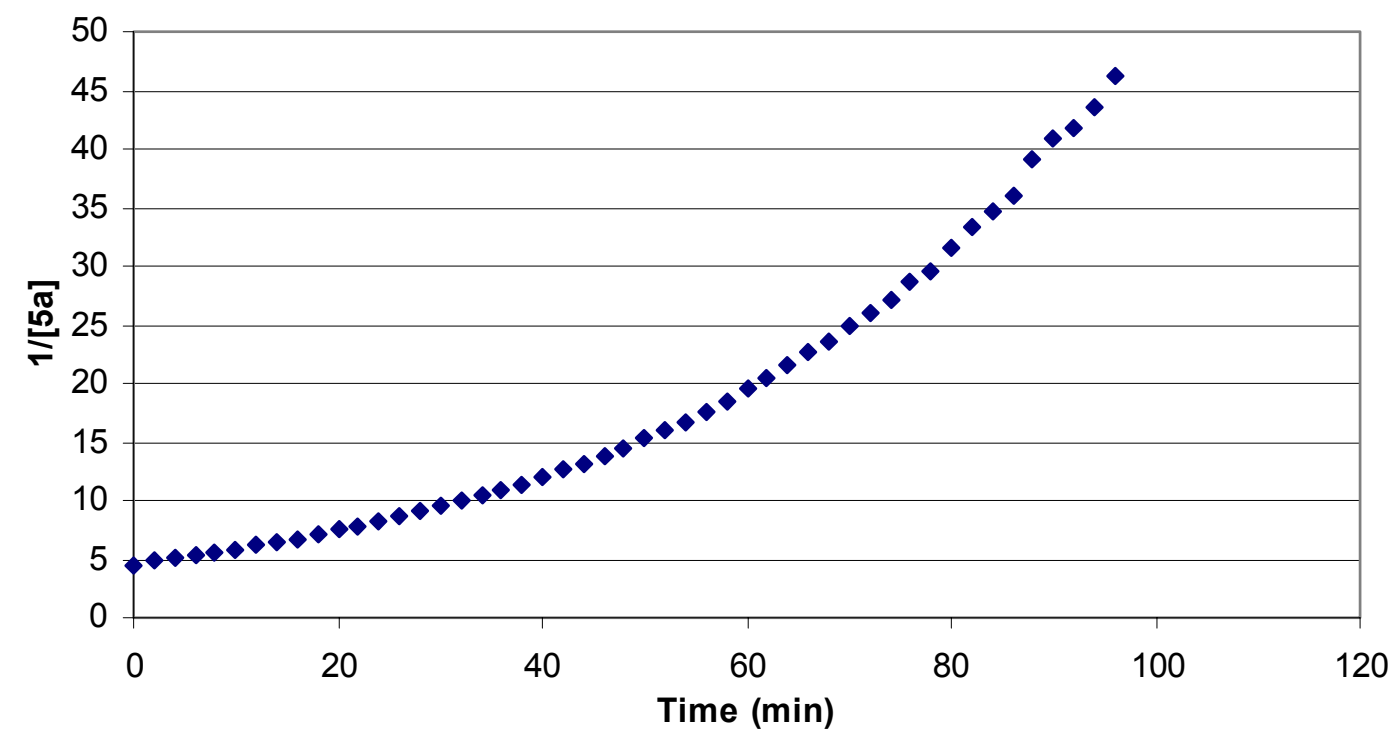

Figure B shows clearly a nonlinear relationship between $1 /[\mathbf{5 a}]$ and time, indicating the reaction is NOT second order in $\mathbf{5 a}$

Figure C. Determination of the order of Dimethyl malonate.

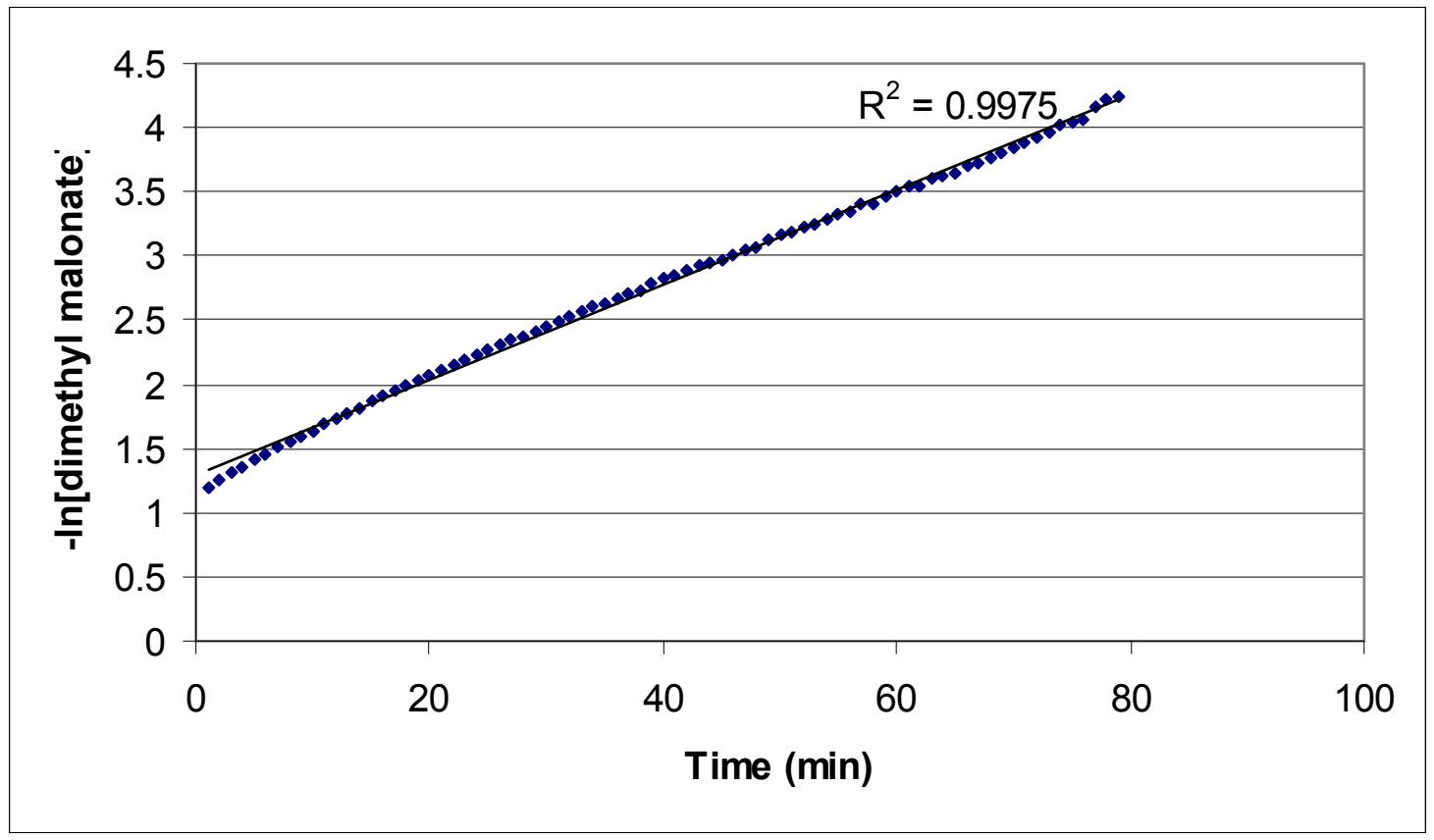

Figure $\mathrm{C}$ shows a linear relationship between $\ln$ [dimethyl malonate] and time, indicating the reaction is first order in dimethyl malonate.

Figure D. Determination of the order of dimethyl malonate. 


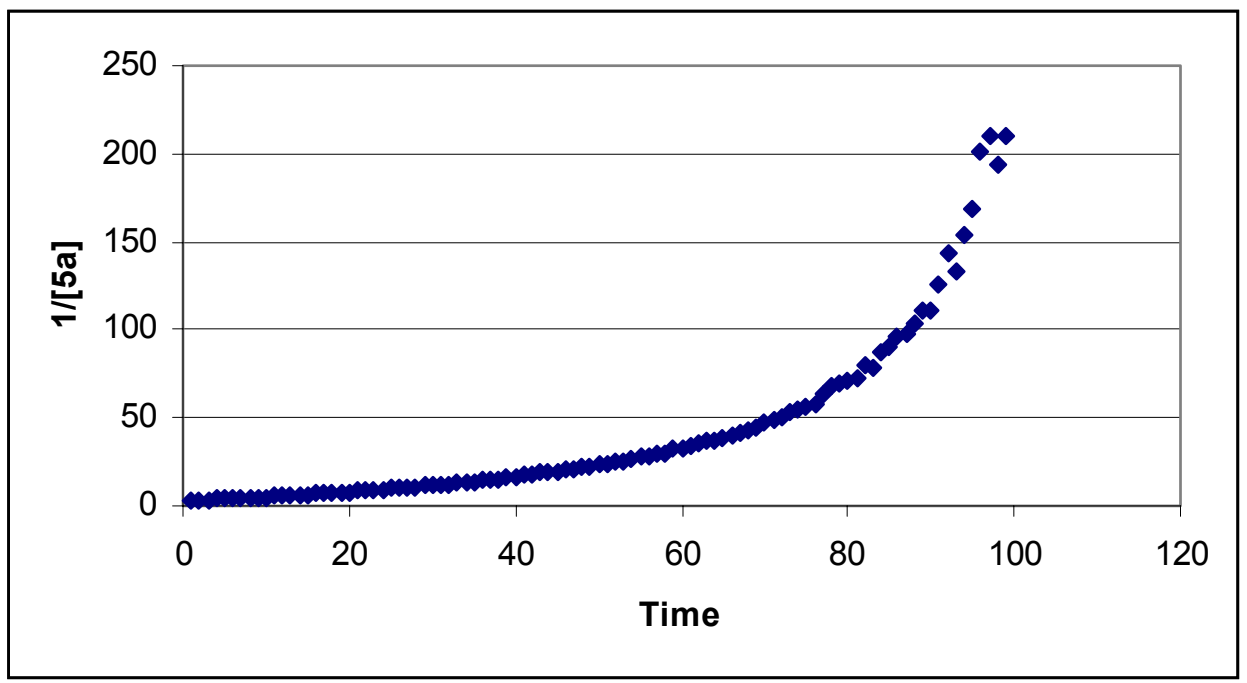

Figure D shows clearly nonlinear relationship between $1 /$ [dimethyl malonate] and time, indicating the reaction is NOT second order in dimethyl malonate

Figure E. Kinetic profiles for the catalyst QD-4a

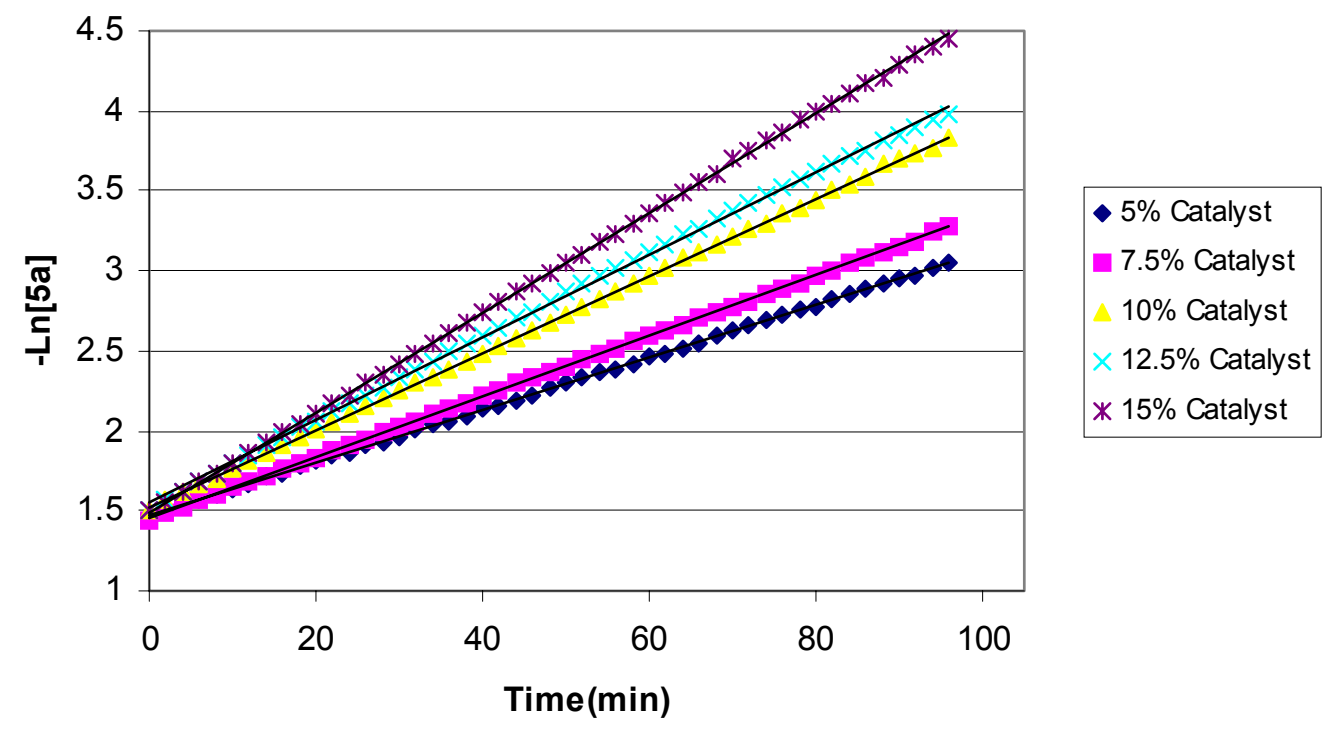

$5.0 \mathrm{~mol} \%$ catalyst

$7.5 \mathrm{~mol} \%$ catalyst

$$
\begin{array}{lll}
5.0 \mathrm{~mol} \% \text { catalyst } & \mathrm{k}_{\mathrm{obs}}=0.01635 & \mathrm{R}^{2}=0.9999 \\
7.5 \mathrm{~mol} \% \text { catalyst } & \mathrm{k}_{\mathrm{obs}}=0.0189 & \mathrm{R}^{2}=0.9999 \\
10.0 \mathrm{~mol} \% \text { catalyst } & \mathrm{k}_{\mathrm{obs}}=0.02414 & \mathrm{R}^{2}=0.9999 \\
12.5 \mathrm{~mol} \% \text { catalyst } & \mathrm{k}_{\mathrm{obs}}=0.0259 & \mathrm{R}^{2}=0.9997
\end{array}
$$$$
15.0 \mathrm{~mol} \% \text { catalyst } \quad \mathrm{k}_{\mathrm{obs}}=0.03115 \quad \mathrm{R}^{2}=0.9999
$$ 
Figure F. Kinetic rate constant $\left(\mathrm{k}_{\mathrm{obs}}\right)$ of different concentration of QD-4a

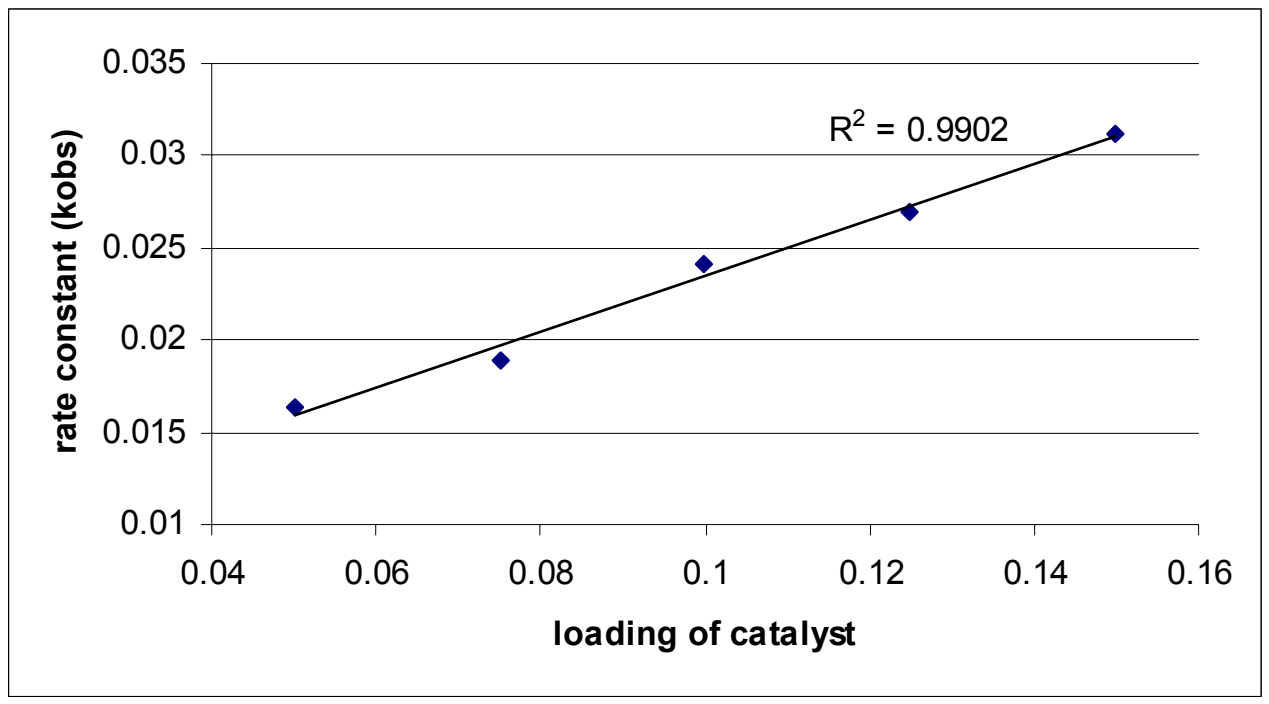

Figure $\mathrm{F}$ shows the linear relationship between the kinetic rate constant $\left(\mathrm{k}_{\mathrm{obs}}\right)$ and the loading of the catalyst, indicating the reaction is first order in catalyst QD-4a.

1 (a) Denmark, S. E.; Marcin, L, R. J. Org. Chem. 1993, 58, 3850-3856. (b) Bulbule, V. J.; Jnaneshwara, G. K.; Deshmukh, R. R.; Borate, H. B.; Deshpande, V. H. Synthetic Comm. 2001, 31, 3623-3626.

${ }^{2}$ Amberg, W.; Bennani, Y.; Chadha, R. K.; Crispino, G. A.; Davis, W. D.; Hartung, J.; Jeong, K.; Ogino, Y.; Shibata, T.; Sharpless, K. B. J. Org. Chem. 1993, 58, 844-849.

${ }^{3}$ Smith, A. B.; Schow, S. R.; Bloom, J. D.; Thompson, A. S.; Winzenberg, K. N. J. Am. Chem. Soc. 1982, 104, 4015-4018.

${ }^{4}$ Ji, J.; Barnes, D. M.; Zhang, J.; King, S. A.; Wittenberger, S. J.; Morton, H. J. Am. Chem. Soc. 1999, 121, 10215-10216. 
HPLC Conditions: Daicel chiralcel OD, Hexane:IPA, 70:30, $0.9 \mathrm{~mL} / \mathrm{min}, \lambda 220 \mathrm{~nm}$
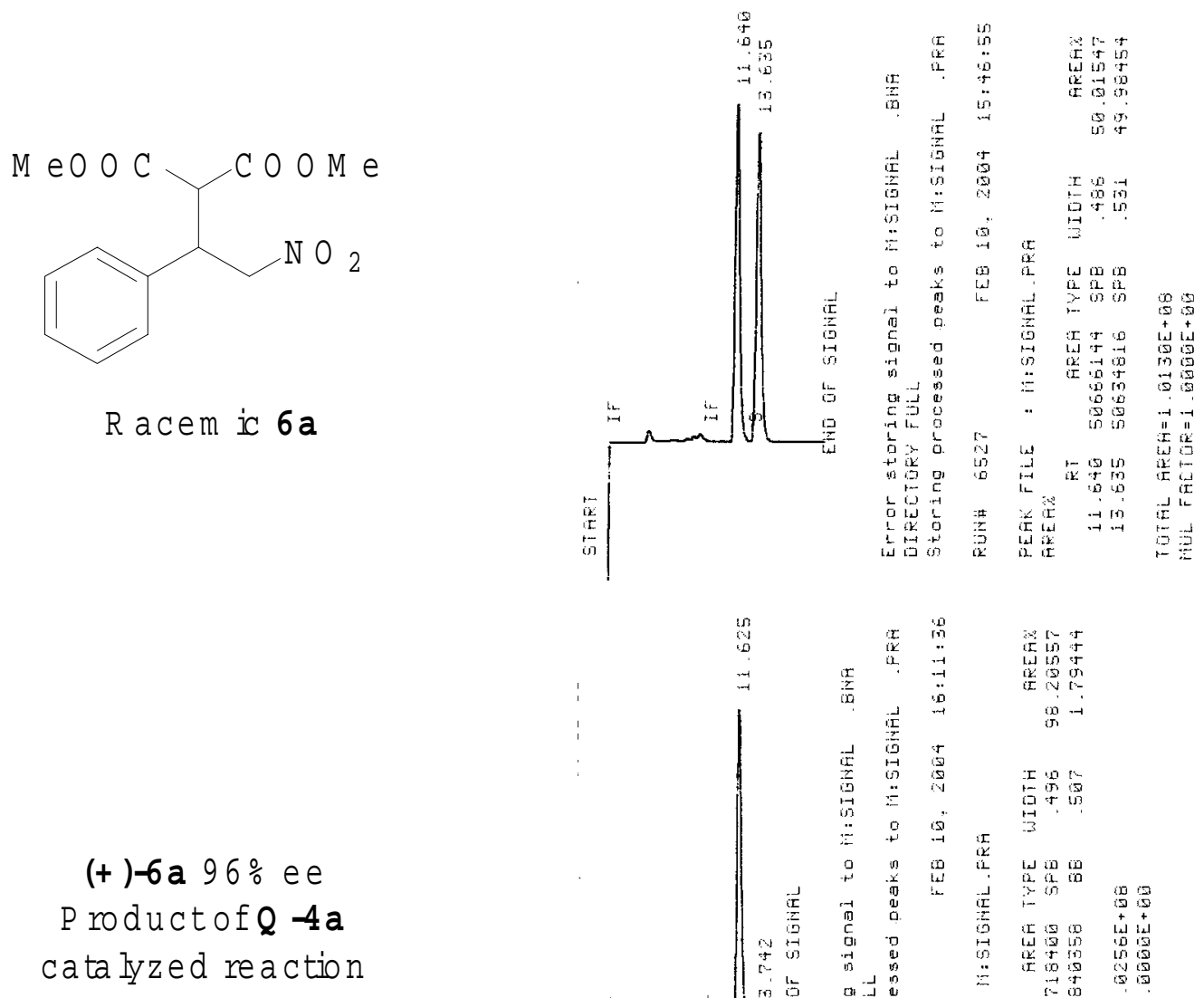

$(-)-6$ a $93 \div$ ee $P$ roduct of $Q D-4 a$ catalyzed reaction

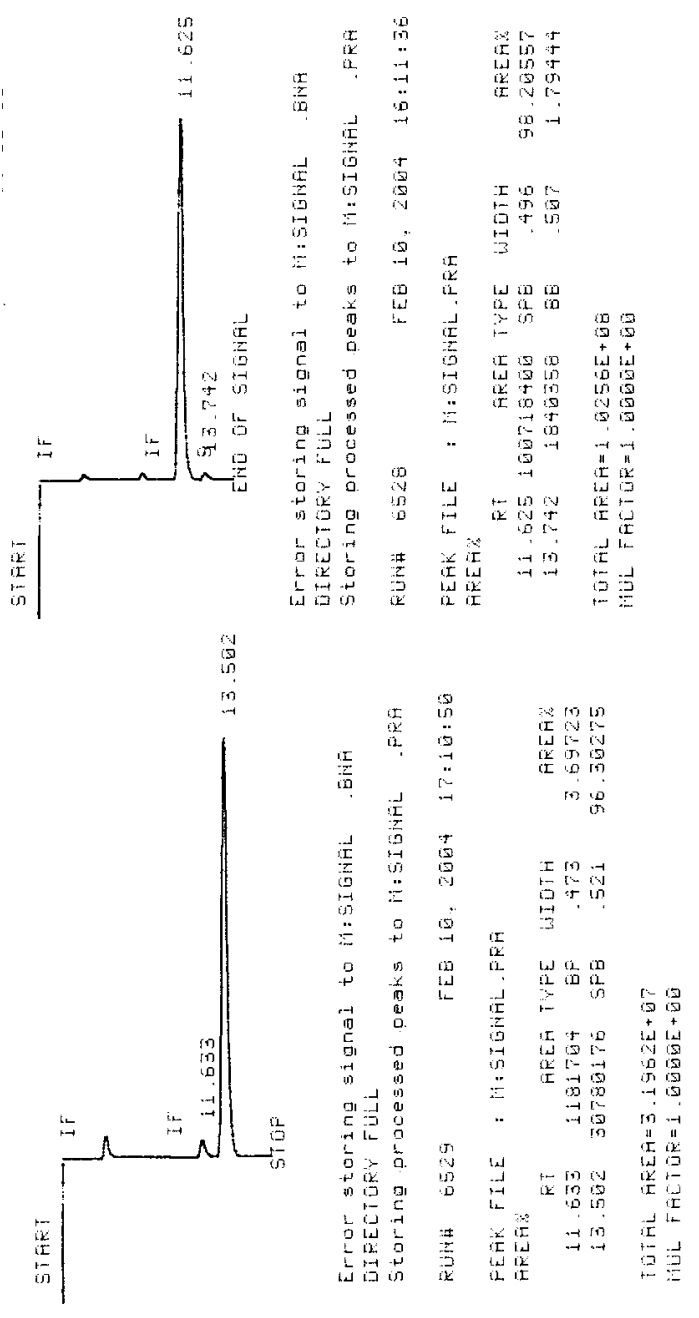


HPLC Conditions: Chiralpak AD, Hexane:IPA, 70:30, $1.0 \mathrm{~mL} / \mathrm{min}, \lambda 220 \mathrm{~nm}$

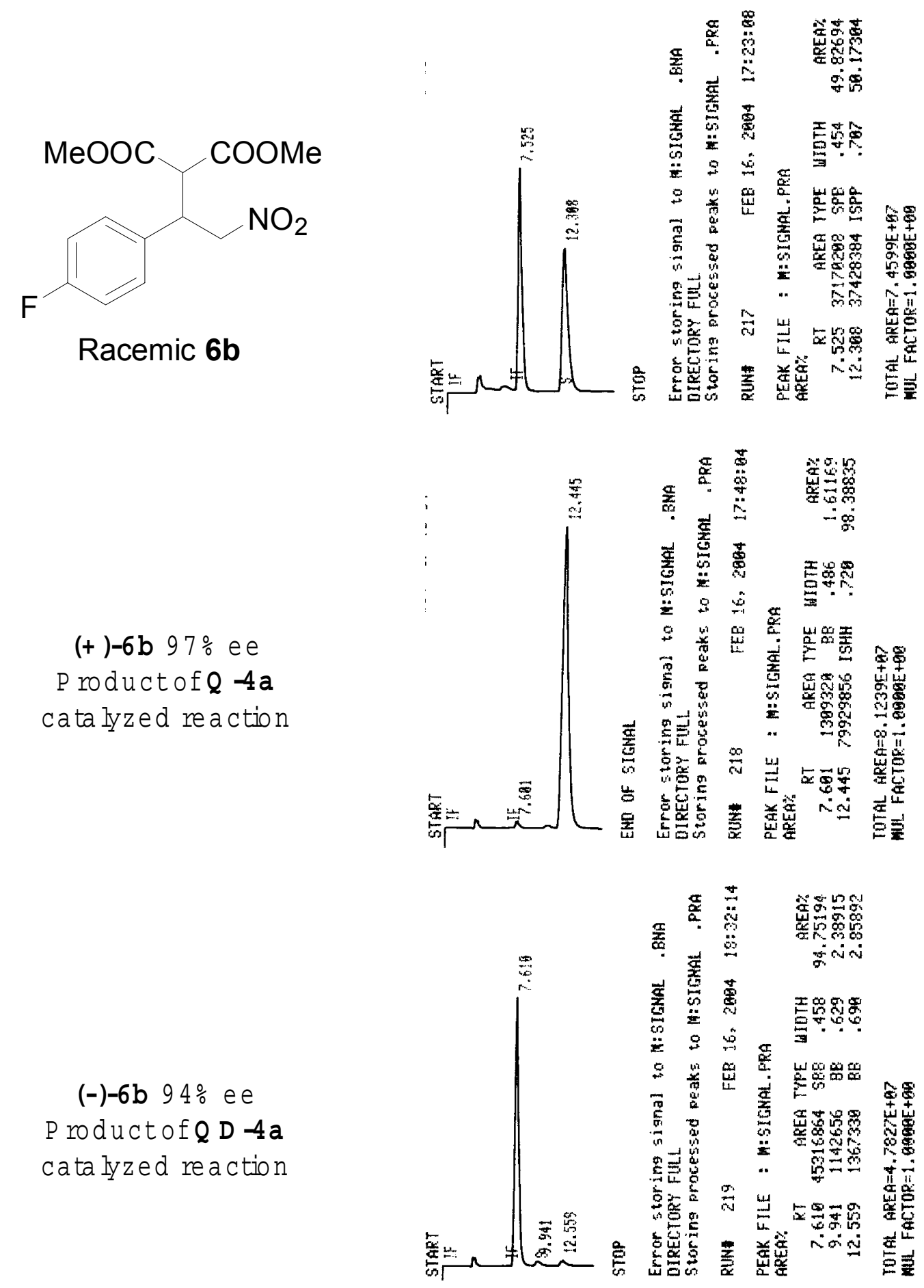


HPLC Conditions: Daicel chiralcel OD, Hexane:IPA, 70:30, $1.0 \mathrm{~mL} / \mathrm{min}, \lambda 220 \mathrm{~nm}$

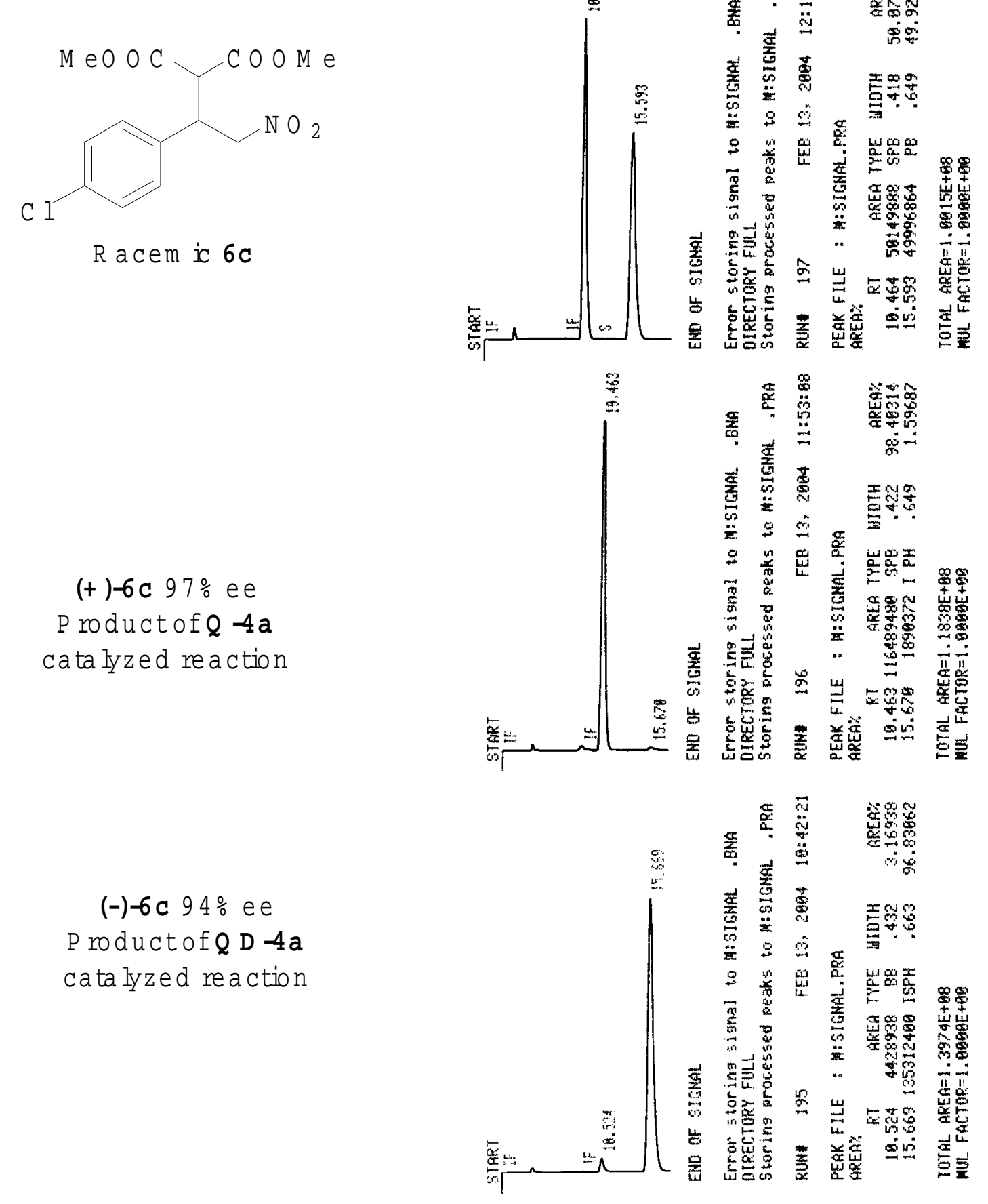


HPLC Conditions: Daicel chiralcel OD, Hexane:IPA, 60:40, $1.0 \mathrm{~mL} / \mathrm{min}, \lambda 220 \mathrm{~nm}$
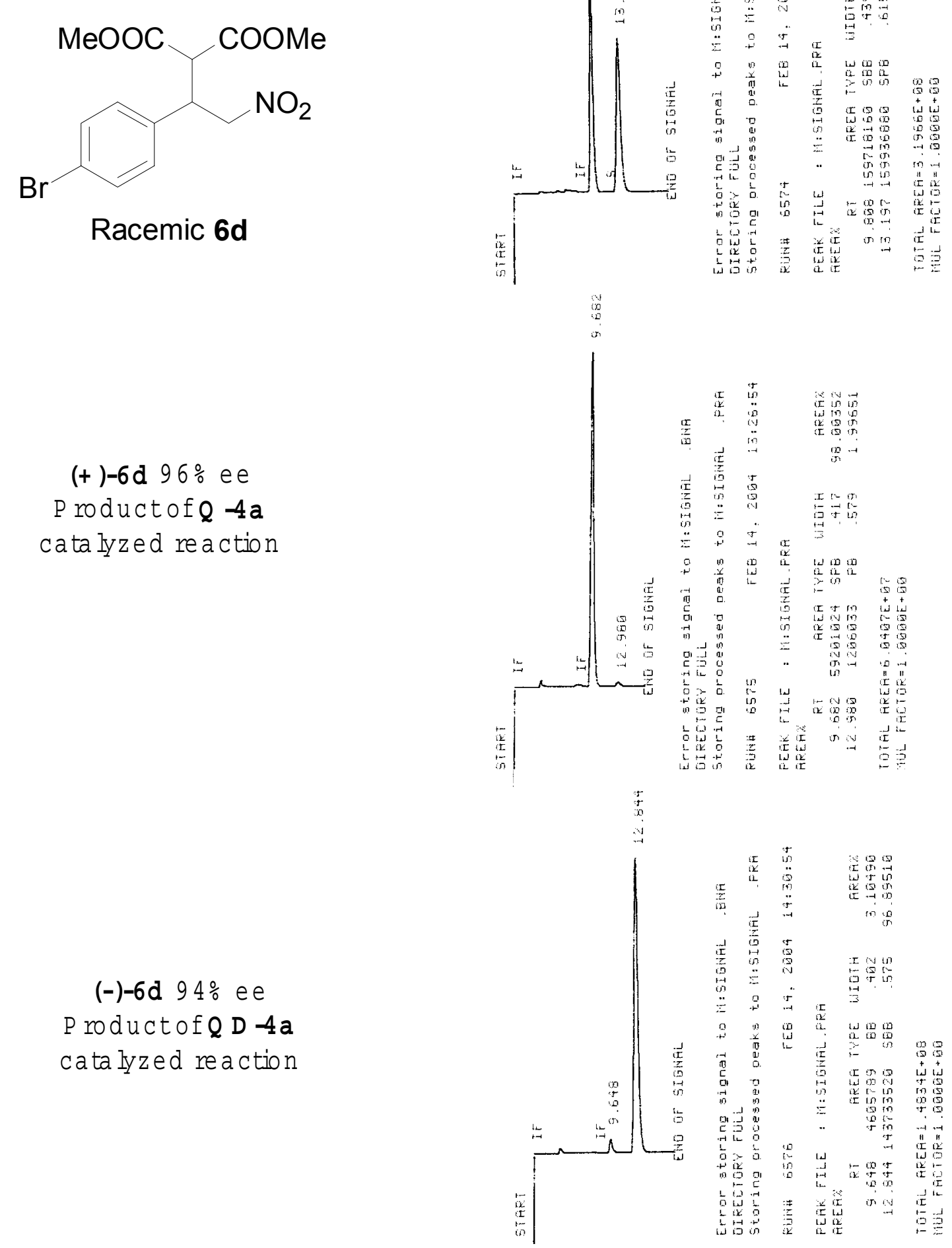

$(-)-6 d 94 \%$ ee P roduct of $Q D-4 a$ cata lyzed reaction

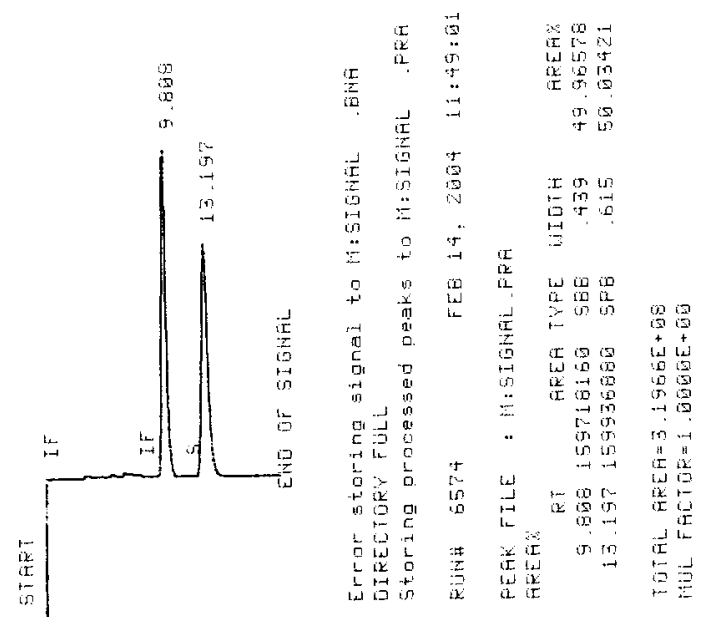


HPLC Conditions: Daicel chiralcel OD, Hexane:IPA, 70:30, $1.0 \mathrm{~mL} / \mathrm{min}, \lambda 220 \mathrm{~nm}$

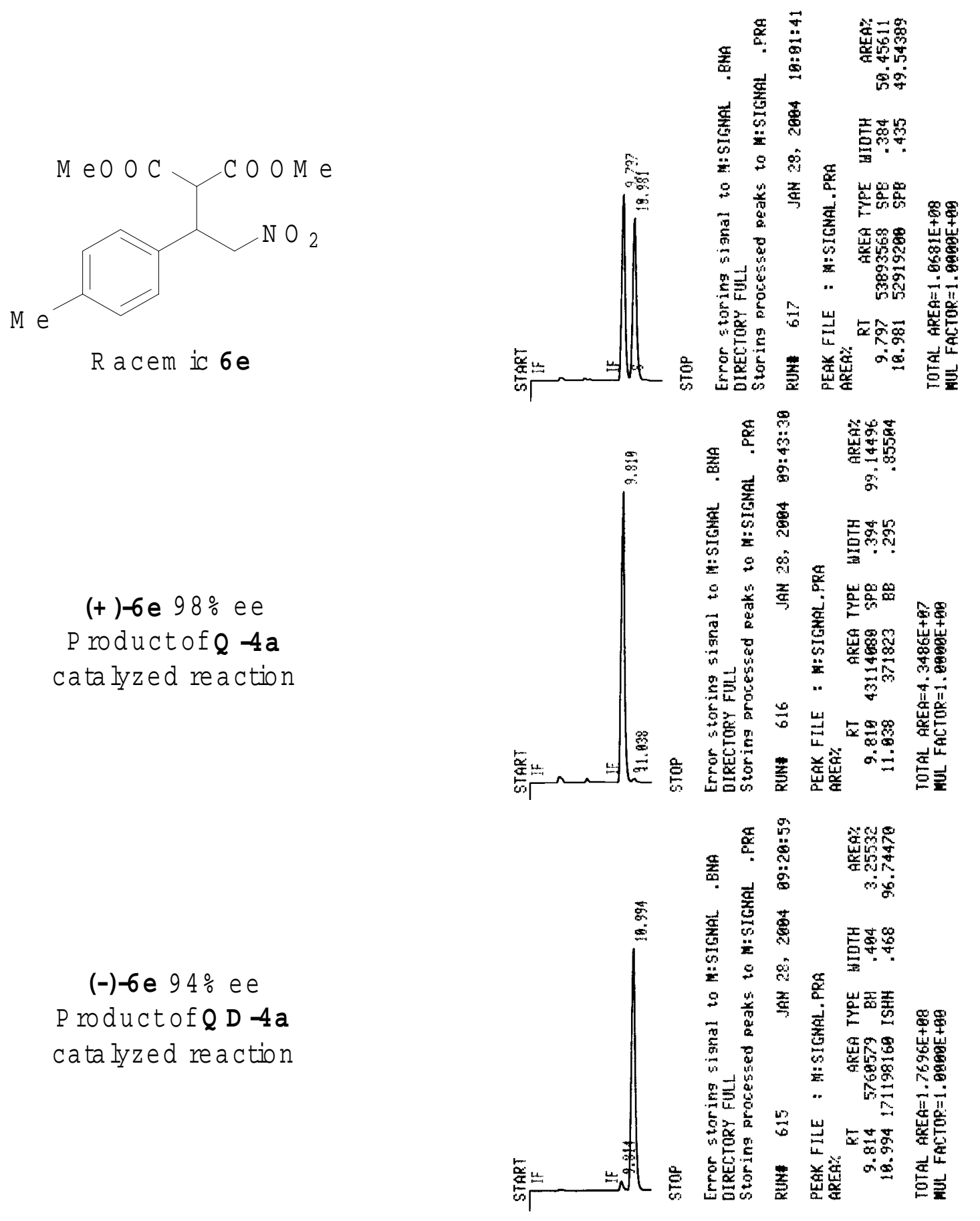


HPLC Conditions: Daicel chiralcel OD, Hexane:IPA, 70:30, $0.9 \mathrm{~mL} / \mathrm{min}, \lambda 220 \mathrm{~nm}$
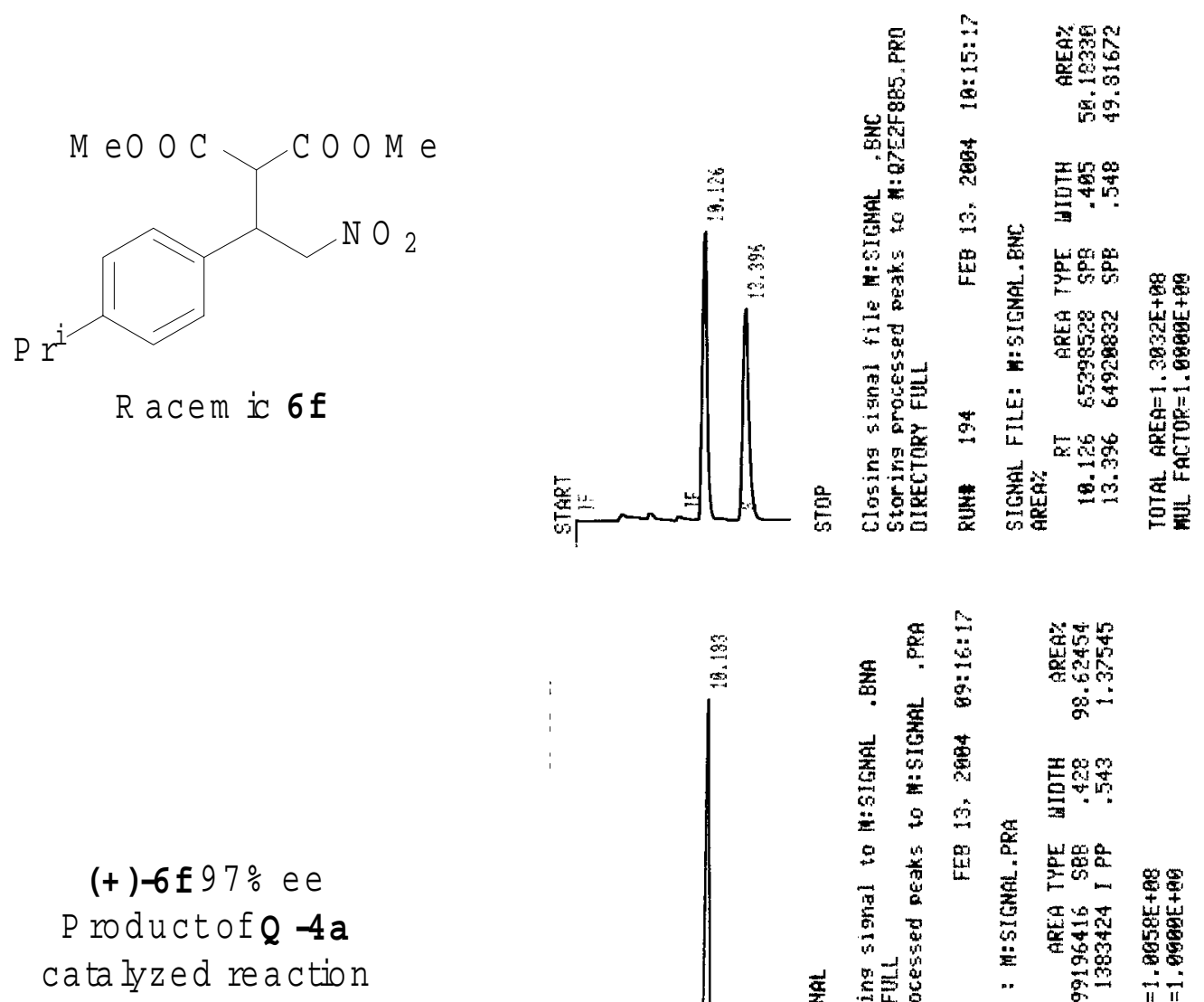

$(-)-6 \mathbf{f} 93 \%$ ee $P$ roduct of $Q D-4 a$ cata lyzed reaction

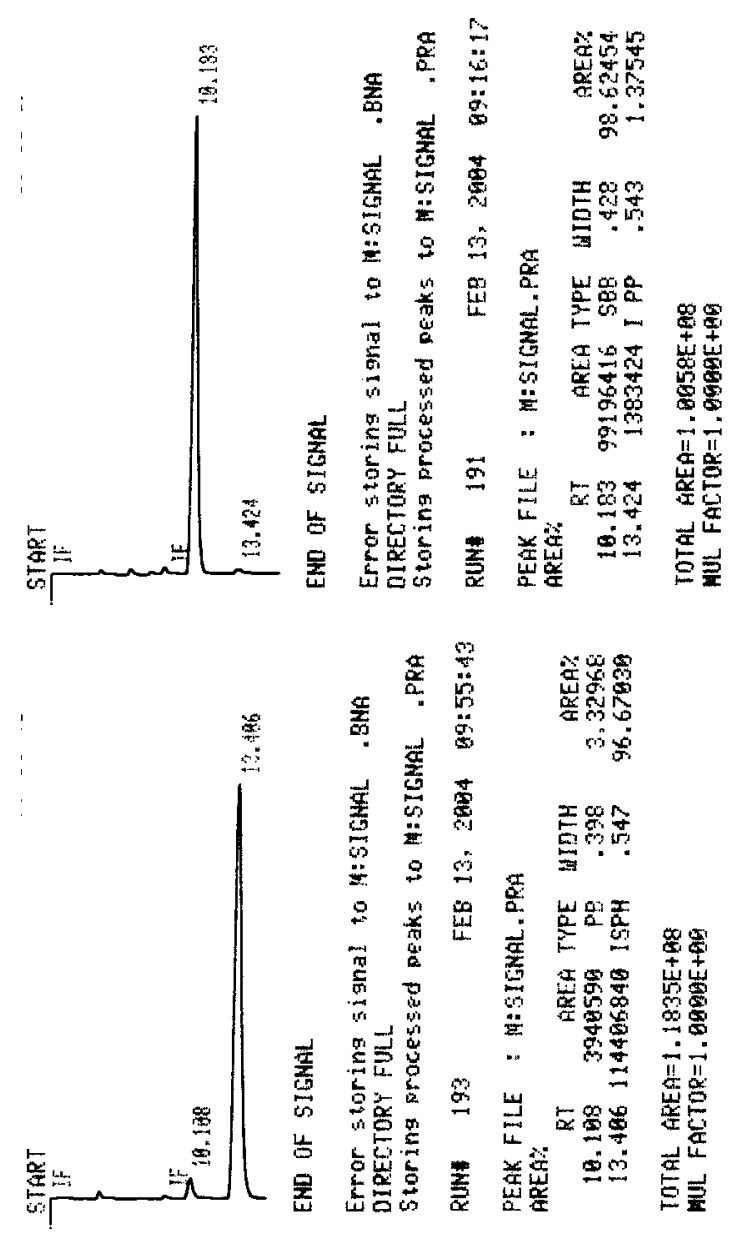


HPLC Conditions: Chiralpak AD, Hexane:IPA, 60:40, $1.0 \mathrm{~mL} / \mathrm{min}, \lambda 220 \mathrm{~nm}$

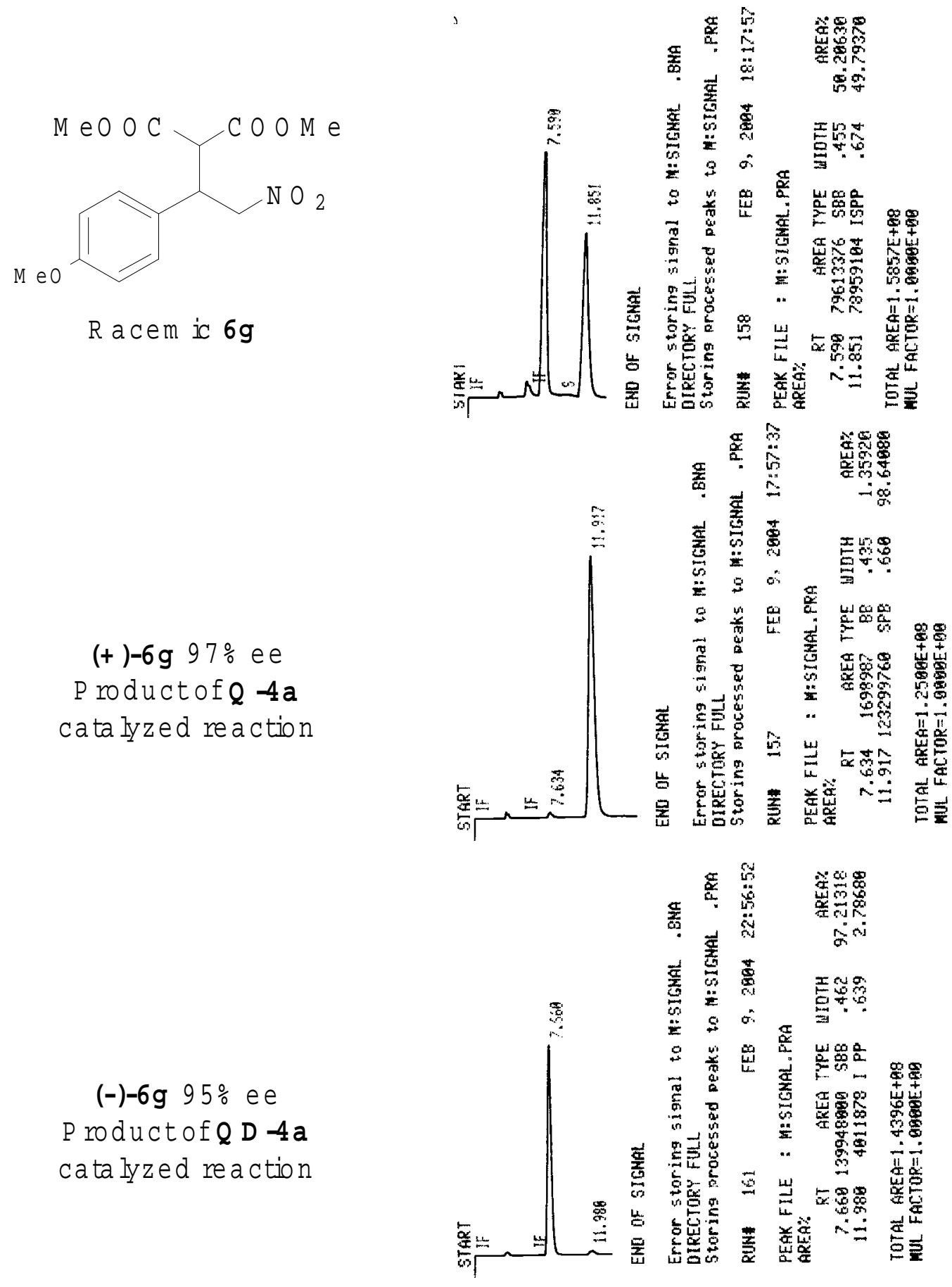


HPLC Conditions: Daicel chiralcel OD, Hexane:IPA, 92:8, $0.9 \mathrm{~mL} / \mathrm{min}, \lambda 220 \mathrm{~nm}$

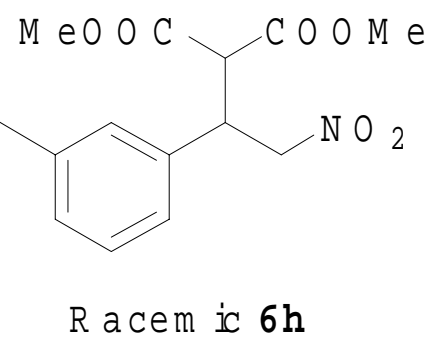

(t) $-6 \mathrm{~h} 97 \%$ ee $P$ roduct of $Q-4$ a catalyzed reaction

$(-)-6 h 93 \div$ ee P roduct of $Q D-4$ a catalyzed reaction
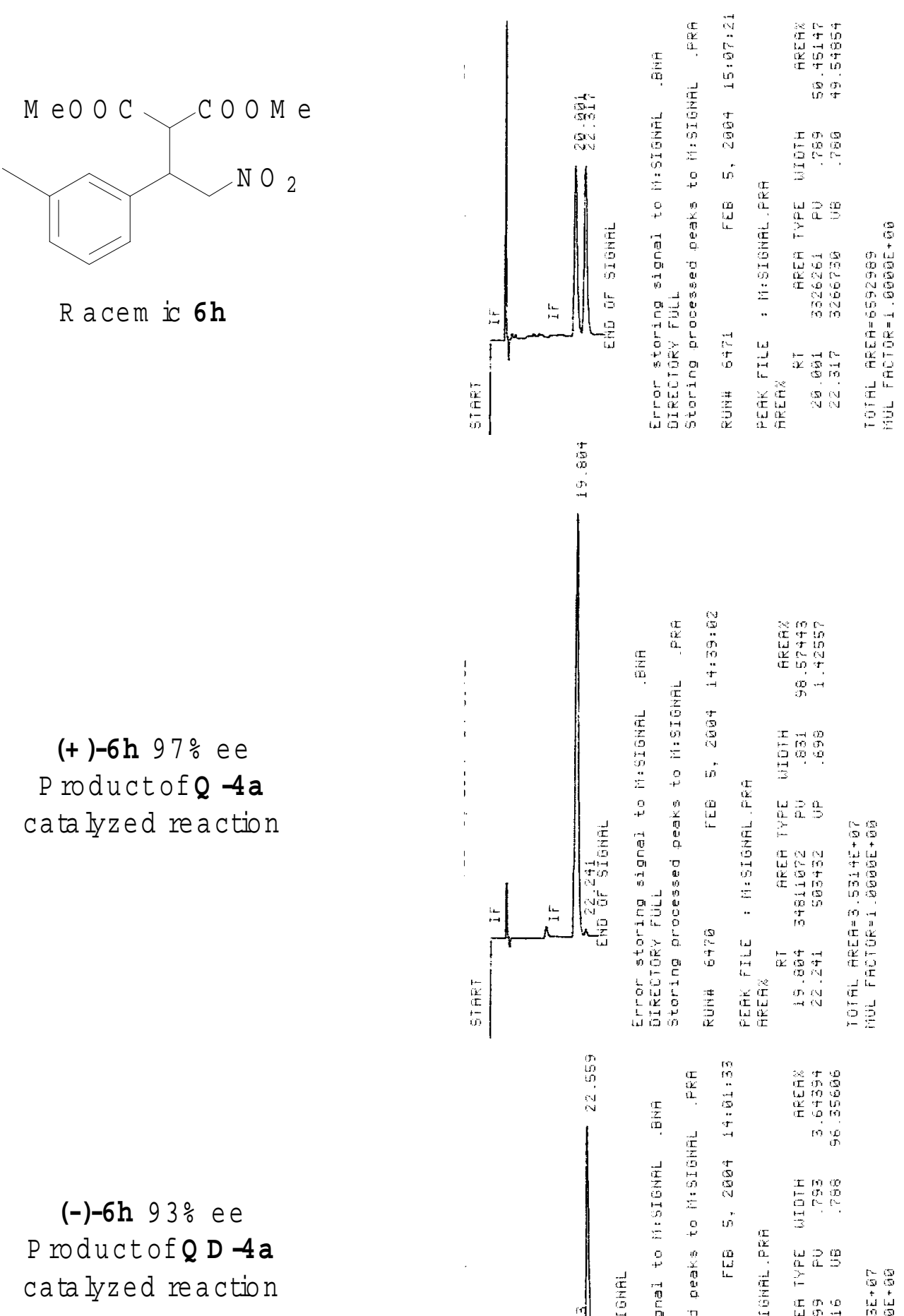
HPLC Conditions: Chiralpak AD, Hexane:IPA, 80:20, $1.0 \mathrm{~mL} / \mathrm{min}, \lambda 220 \mathrm{~nm}$

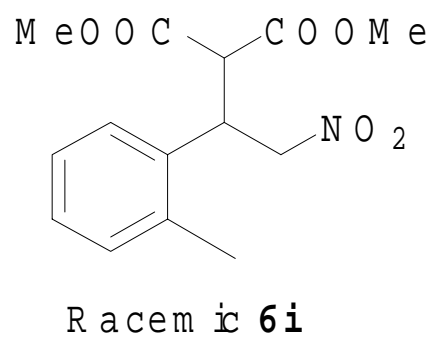

$(-)-6$ i $98 \%$ ee $P$ roduct of $Q-4 a$ catalyzed reaction

(t) -6 i $96 \%$ ee $P$ roduct of $Q D-4 a$ catalyzed reaction
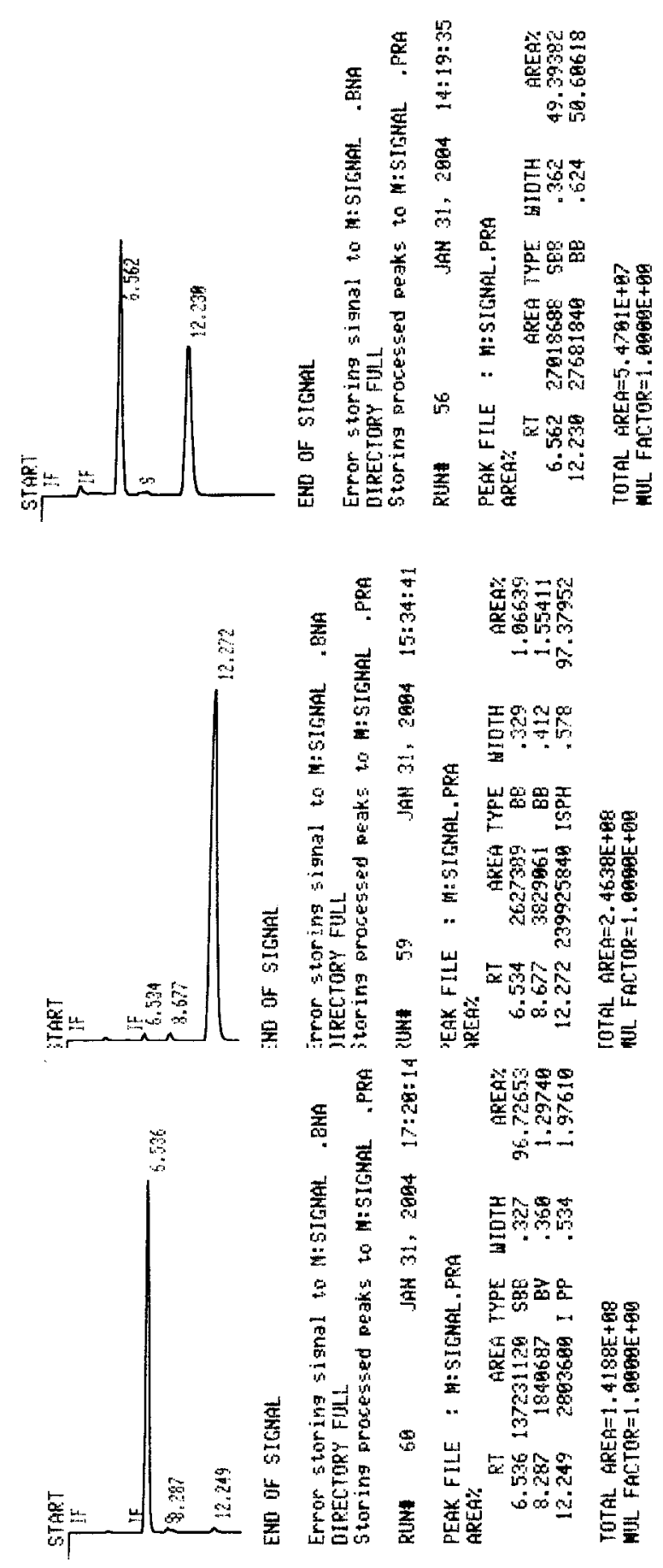
HPLC Conditions: Chiralpak AD, Hexane:IPA, 80:20, $1.0 \mathrm{~mL} / \mathrm{min}, \lambda 220 \mathrm{~nm}$
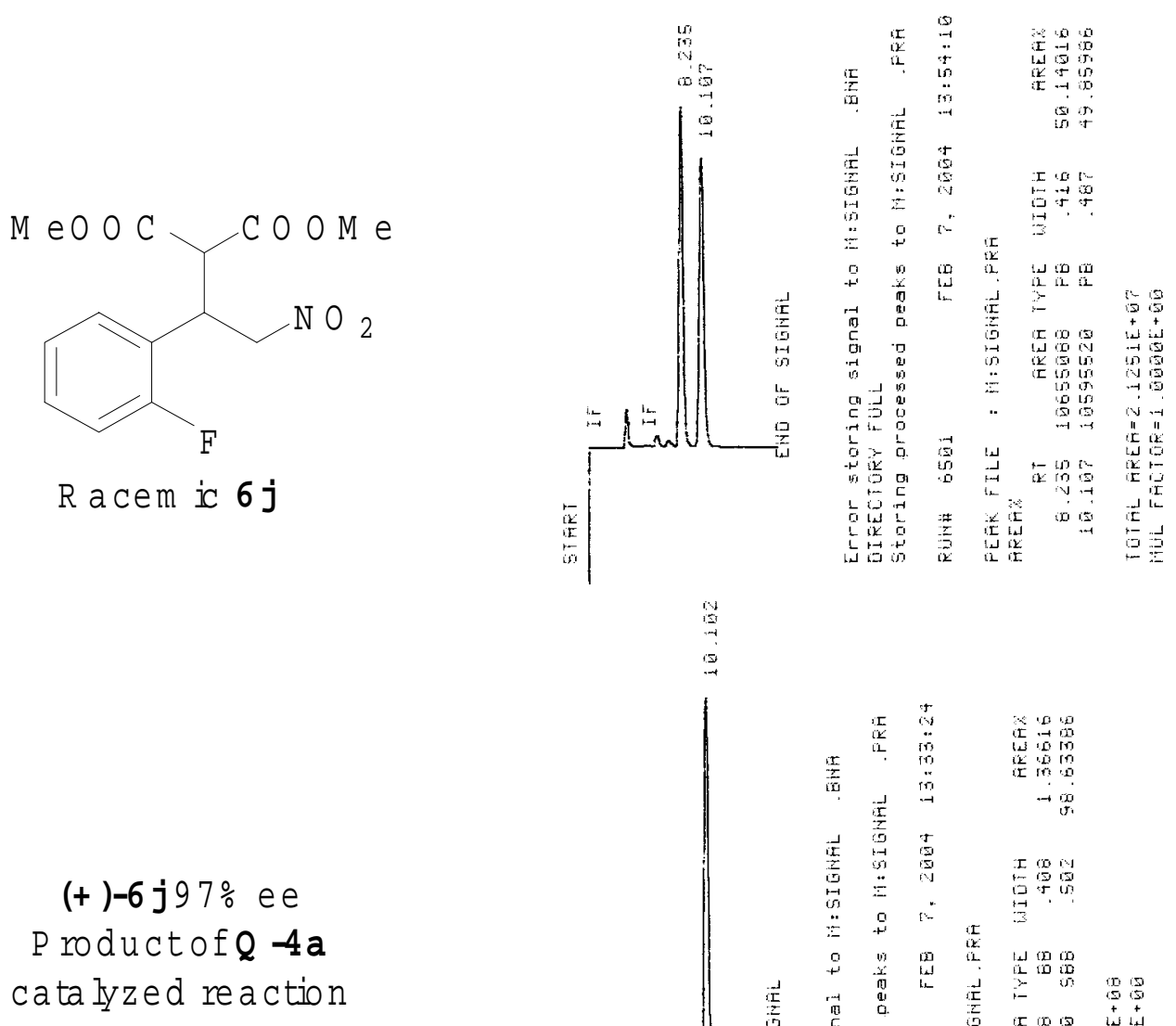

$(-)-6 j 95 \%$ ee $P$ roduct of $Q D-4 a$ catalyzed reaction
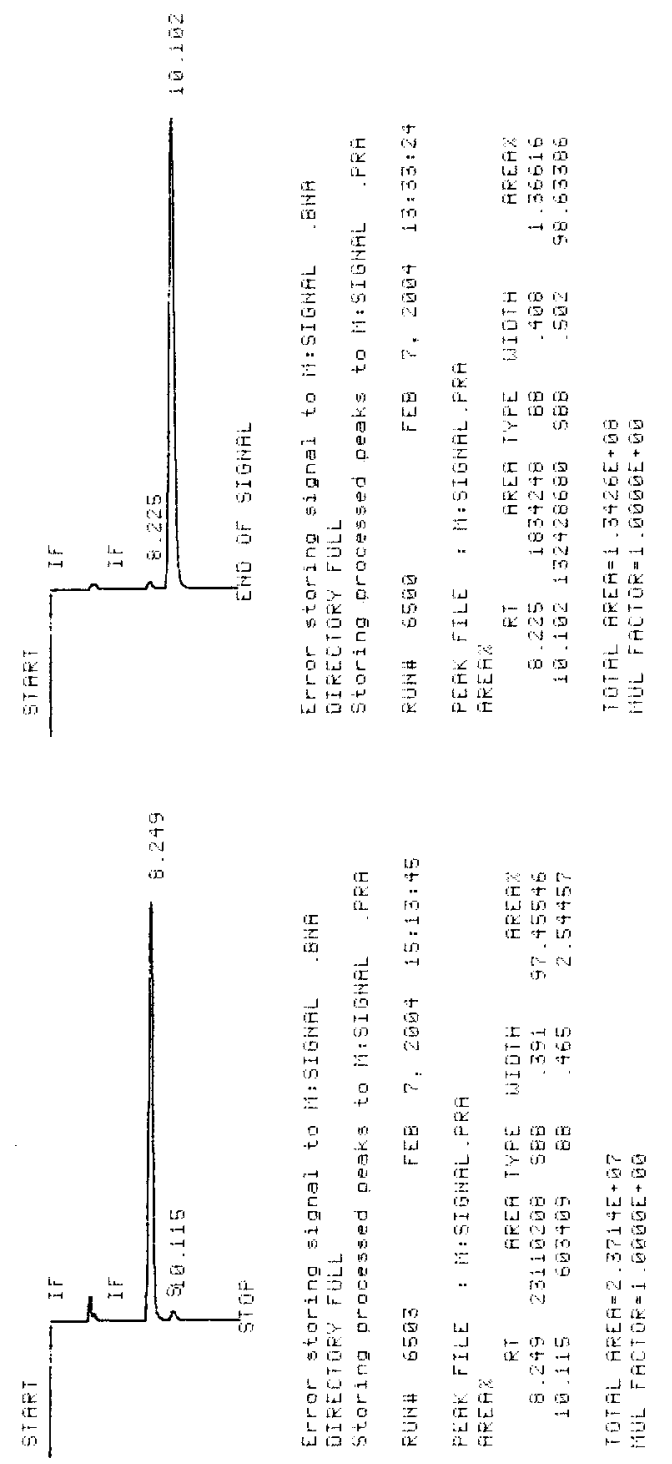
HPLC Conditions: Daicel chiralcel OD, Hexane:IPA, 50:50, 0.9 mL/min, $\lambda 220 \mathrm{~nm}$

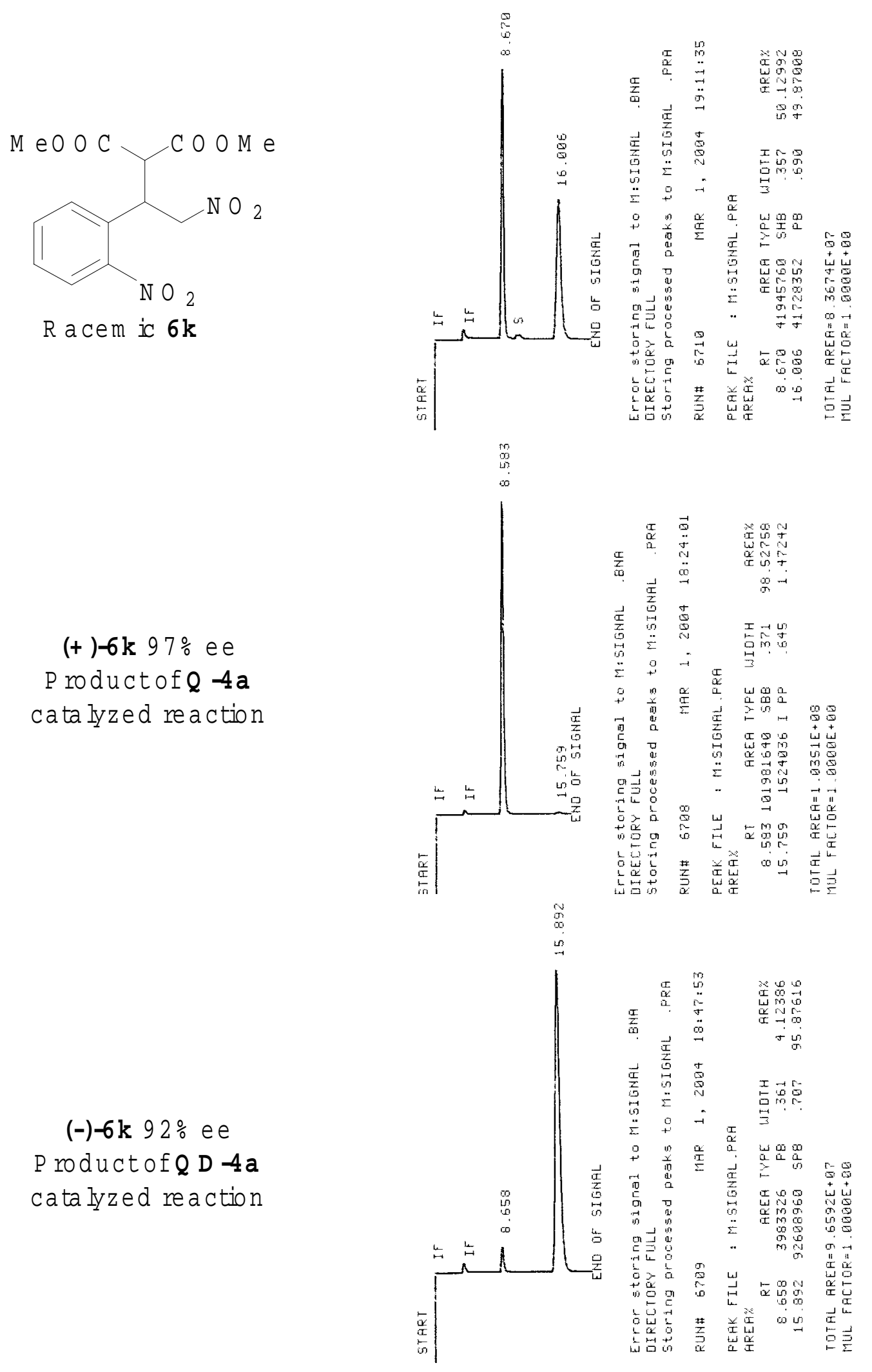


HPLC Conditions: Chiralpak AD, Hexane:IPA, 80:20, $1.0 \mathrm{~mL} / \mathrm{min}, \lambda 280 \mathrm{~nm}$

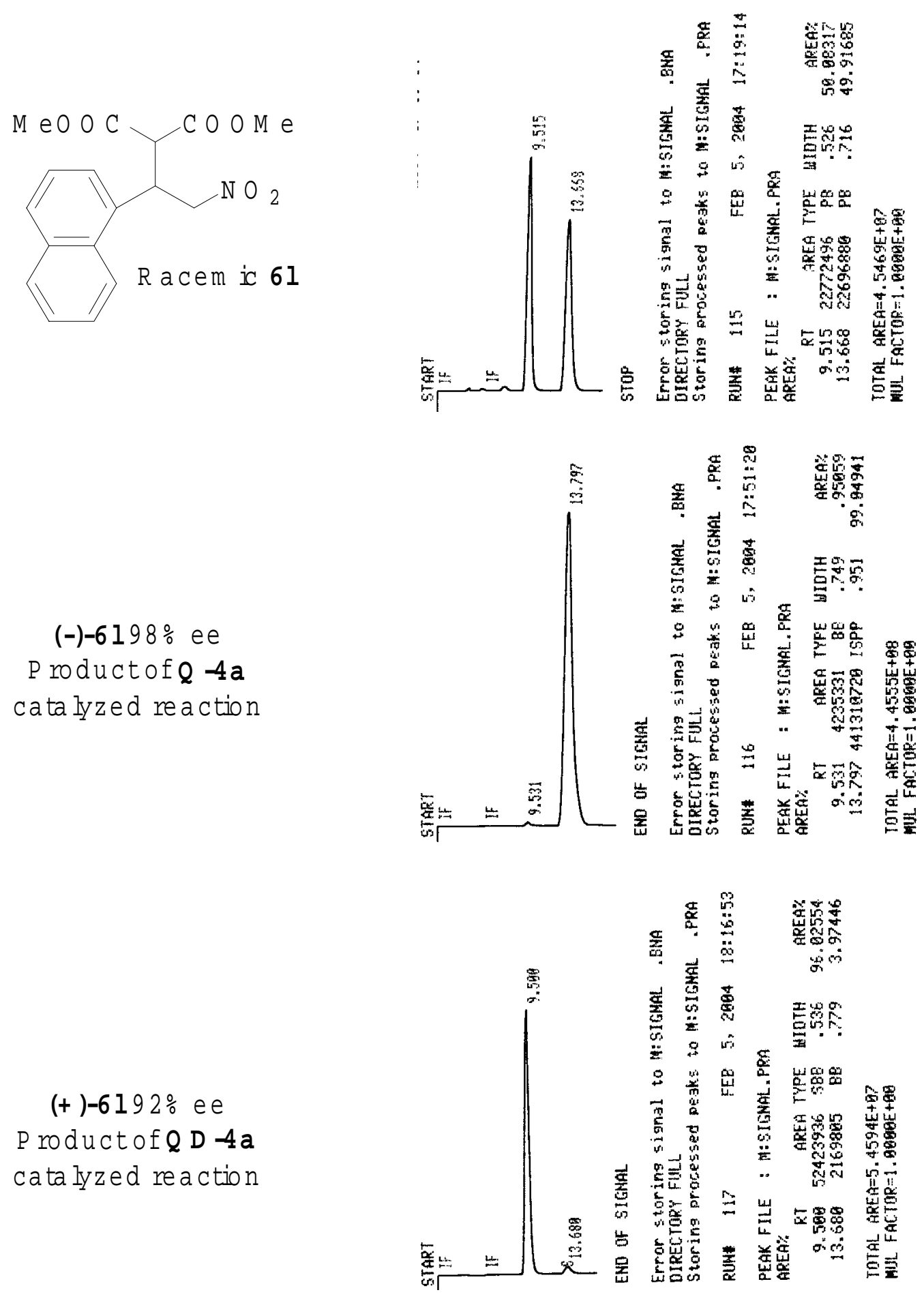


HPLC Conditions: Chiralpak AD, Hexane:IPA, 80:20, $1.0 \mathrm{~mL} / \mathrm{min}, \lambda 220 \mathrm{~nm}$

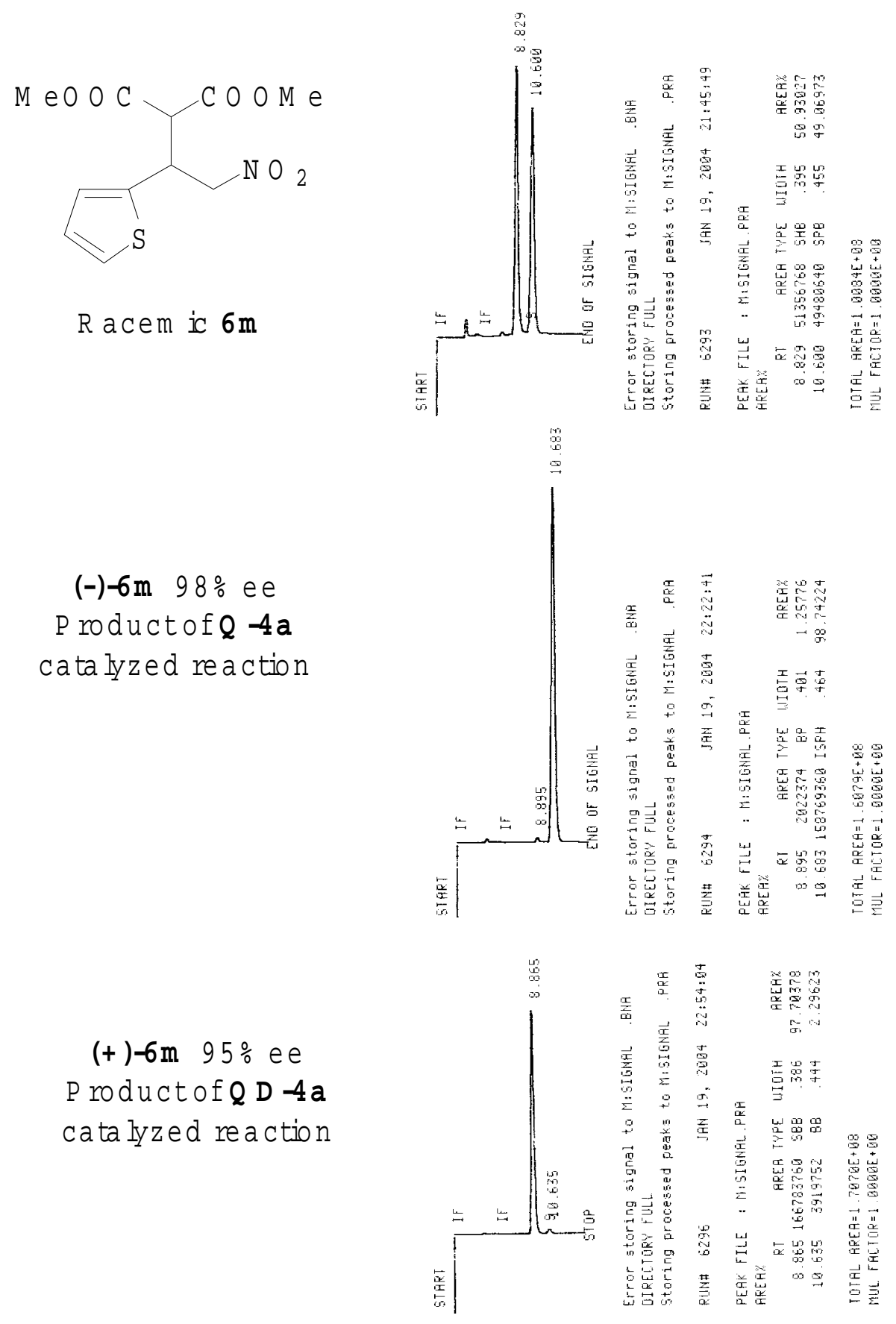


HPLC Conditions: Daicel chiralcel OD, Hexane:IPA, 60:40, $1.0 \mathrm{~mL} / \mathrm{min}, \lambda 220 \mathrm{~nm}$

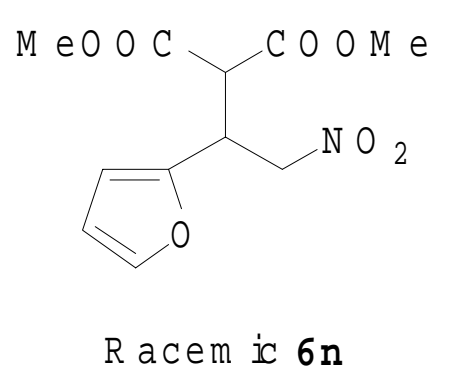

$(-)-6 n 98 \%$ ee $P$ roduct of $Q-4 a$ cata lyzed reaction

(t) $-6 \mathrm{n} 96 \%$ ee

$P$ roduct of $Q D-4 a$

cata lyzed reaction
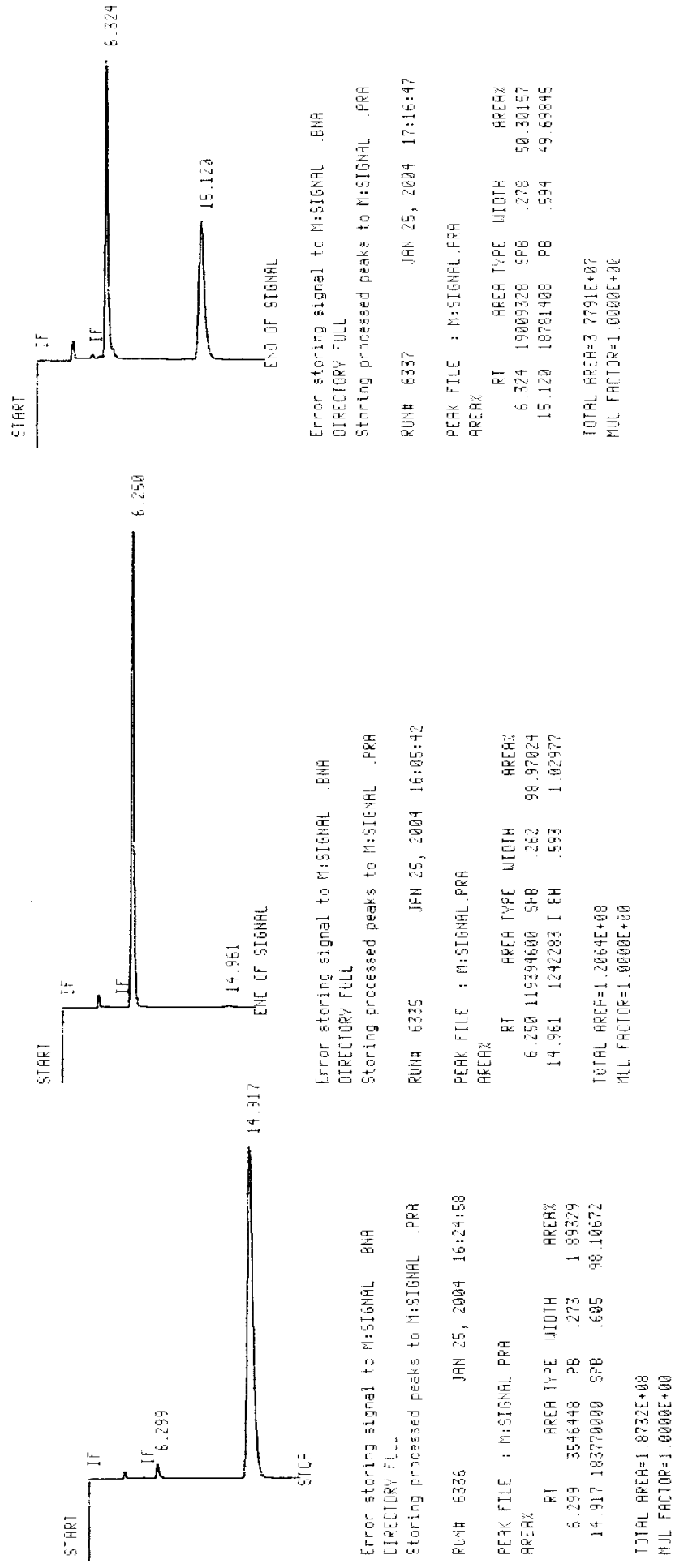
HPLC Conditions: Chiralpak AD, Hexane:IPA, 60:40, 1.0 mL/min, $\lambda 220 \mathrm{~nm}$
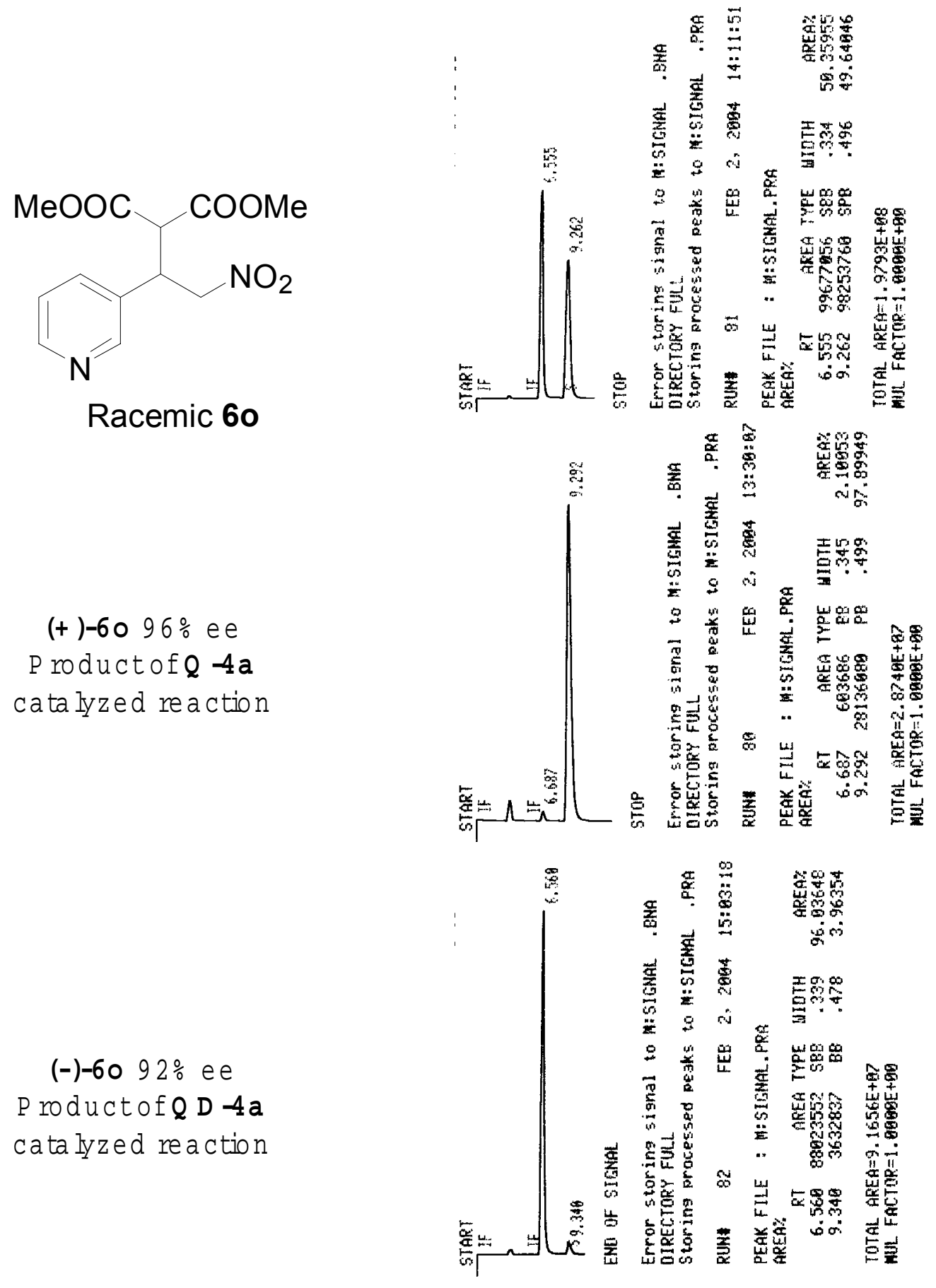
HPLC Conditions: Daicel chiralcel OD, Hexane:IPA, 90:10, $1.0 \mathrm{~mL} / \mathrm{min}, \lambda 215 \mathrm{~nm}$
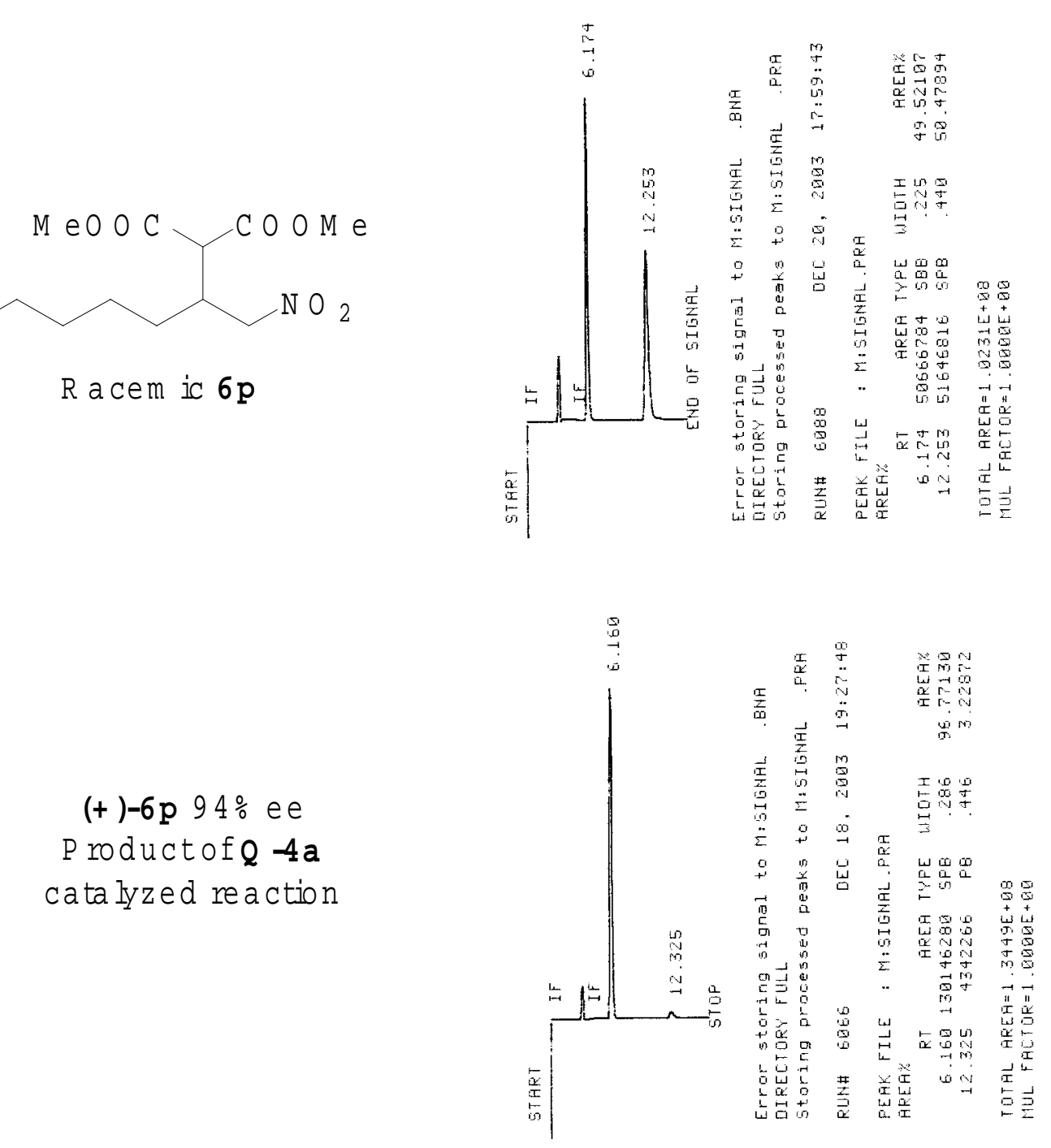

(-) -6p $91 \%$ ee $P$ roduct of $Q D-4 a$ catalyzed reaction

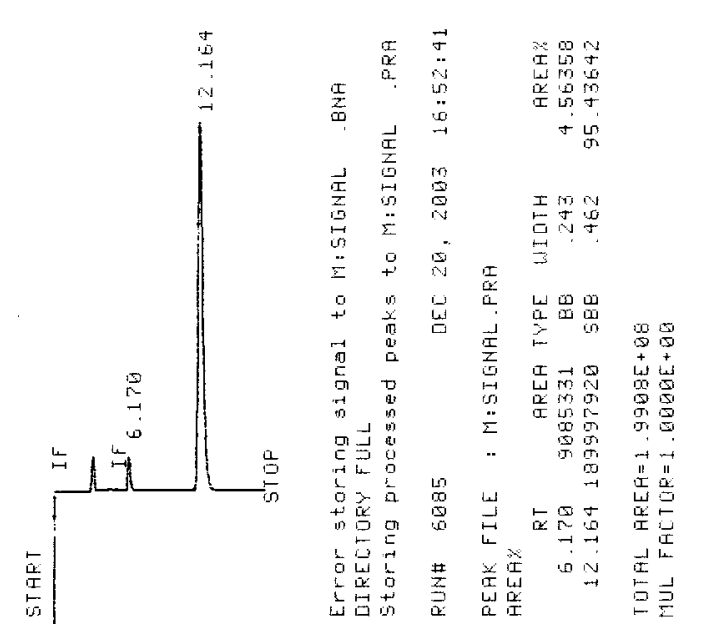


HPLC Conditions: Daicel chiralcel OD, Hexane:IPA, 90:10, $1.0 \mathrm{~mL} / \mathrm{min}, \lambda 215 \mathrm{~nm}$

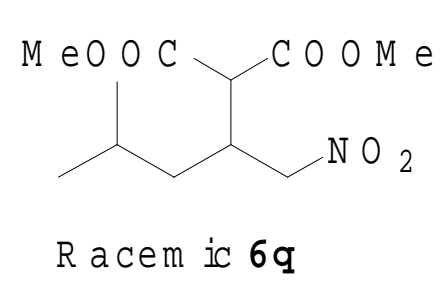

$(+)-6$ q $94 \%$ ee
P roduct of $\mathbf{Q}-4$ a
cata lyzed reaction

$(-)-6 q 92 \%$ ee

$P$ roduct of $Q D-4 a$

cata lyzed reaction

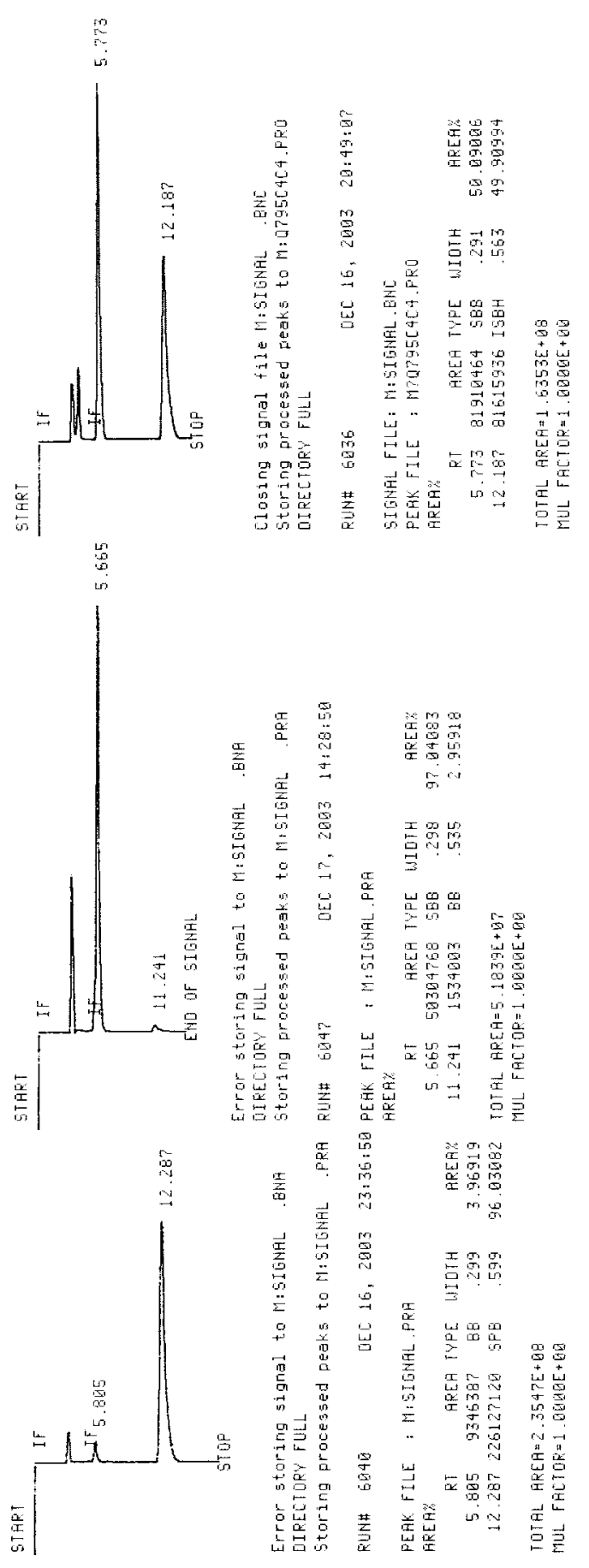


HPLC Conditions: Daicel chiralcel OD, Hexane:IPA, 90:10, $1.0 \mathrm{~mL} / \mathrm{min}, \lambda 215 \mathrm{~nm}$

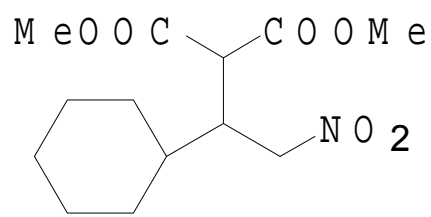

Racem ic $6 r$

(t) -6 r $94 \%$ ee $P$ roduct of $Q-4 a$ catalyzed reaction

$(-)-6 r 91 \%$ ee $P$ roduct of $Q D-4 a$ cata lyzed re a ction
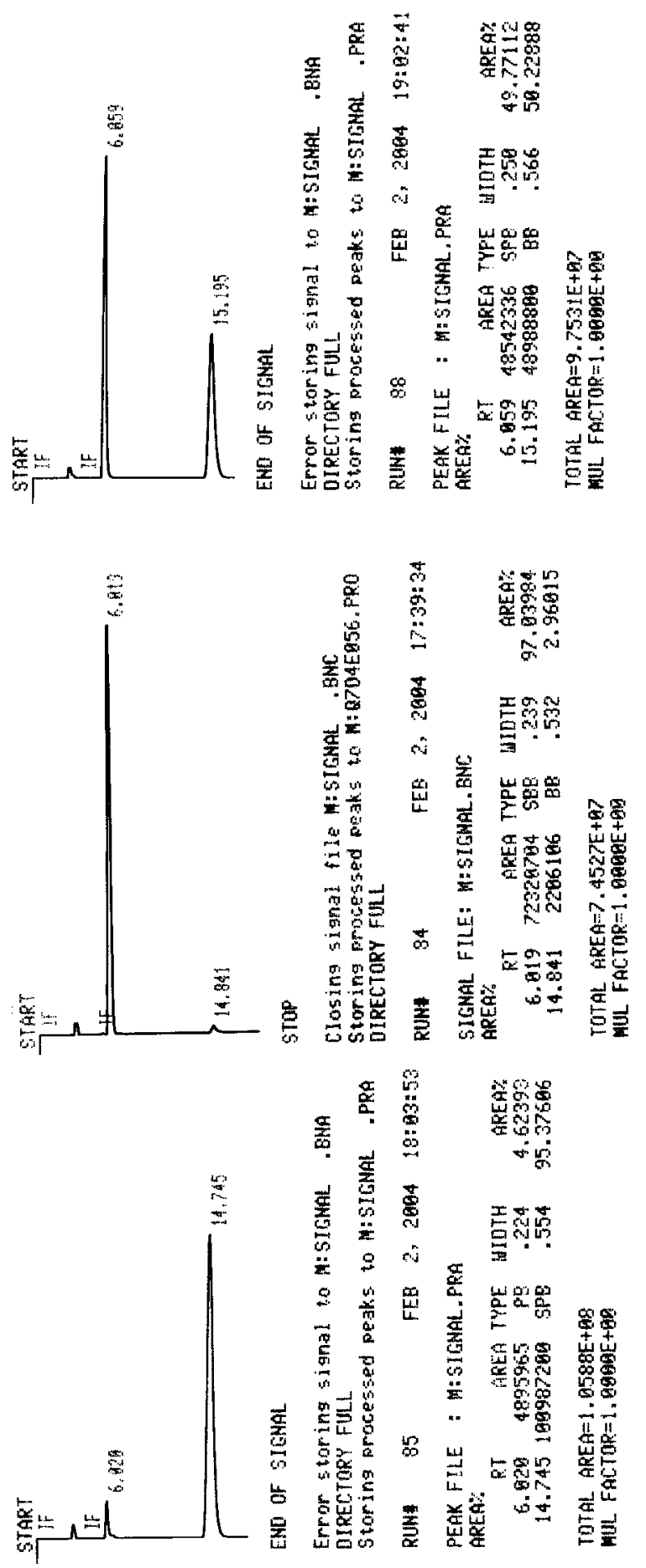
HPLC Conditions: Daicel chiralpak AD, Hexane:Ethanol, 90:10, $0.8 \mathrm{~mL} / \mathrm{min}, \lambda 210$ nm
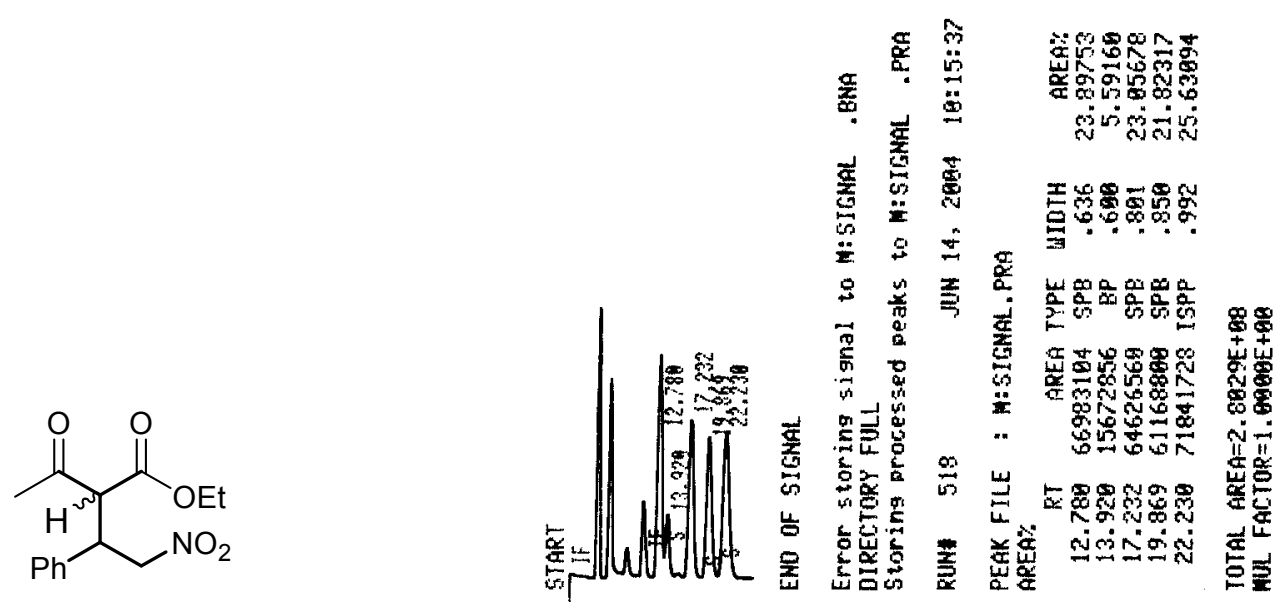

Racemic 8

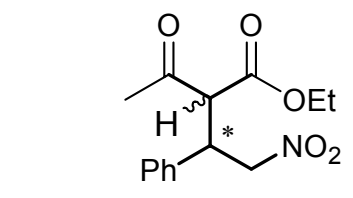

$891 \%$ ee as an around 1:1 mixture of diastereomer product of $\mathbf{Q}-\mathbf{4 a}$ catalyzed reaction

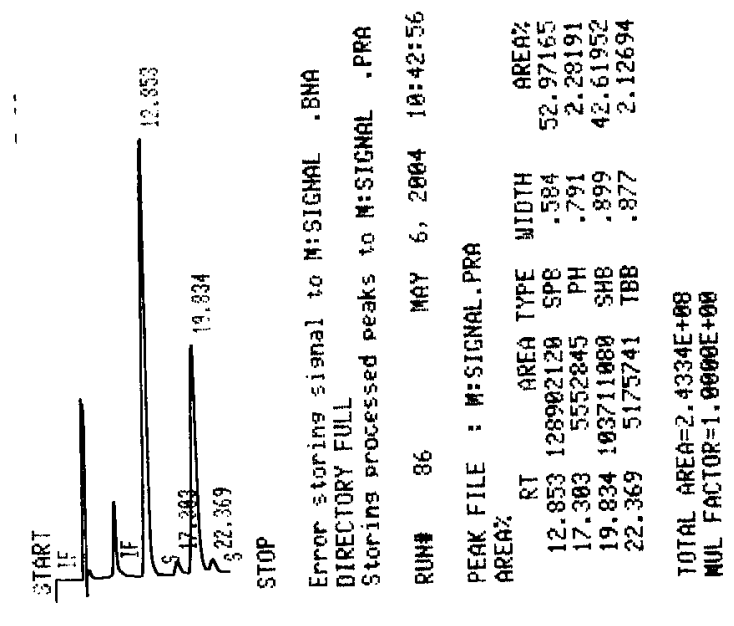

